\title{
ASYMPTOTIC STABILITY OF STATIONARY SOLUTIONS TO THE COMPRESSIBLE EULER-MAXWELL EQUATIONS
}

\author{
QINGQING LIU AND CHANGJIANG ZHU*
}

\begin{abstract}
In this paper, we are concerned with the compressible Euler-Maxwell equations with a nonconstant background density (e.g. of ions) in three dimensional space. There exist stationary solutions when the background density is a small perturbation of a positive constant state. We first show the asymptotic stability of solutions to the Cauchy problem near the stationary state provided that the initial perturbation is sufficiently small. Moreover the convergence rates are obtained by combining the $L^{p}-L^{q}$ estimates for the linearized equations with time-weighted estimate.
\end{abstract}

\section{CONTENTS}

1. Introduction

2. Existence of stationary solution

3. Stability of stationary solution

3.1. Reformulation of the problem

3.2. a priori estimates

4. Decay in time for the non-linear system

References

\section{INTRODUCTION}

The dynamics of two separate compressible fluids of ions and electrons interacting with their self-consistent electromagnetic field in plasma physics can be described by the compressible 2-fluid Euler-Maxwell equations [1, 22. In this paper, we consider the following one-fluid compressible Euler-Maxwell system when the background density $n_{b}$ is a function of spatial variable and the electrons flow is isentropic (see [5, 25, 26] when $n_{b}=$ const.), taking the form of

$$
\left\{\begin{array}{l}
\partial_{t} n+\nabla \cdot(n u)=0, \\
\partial_{t} u+u \cdot \nabla u+\frac{1}{n} \nabla p(n)=-(E+u \times B)-\nu u, \\
\partial_{t} E-\nabla \times B=n u, \\
\partial_{t} B+\nabla \times E=0, \\
\nabla \cdot E=n_{b}(x)-n, \quad \nabla \cdot B=0 .
\end{array}\right.
$$

Here, $n=n(t, x) \geq 0$ is the electron density, $u=u(t, x) \in \mathbb{R}^{3}$ is the electron velocity, $E=E(t, x) \in \mathbb{R}^{3}, B=B(t, x) \in \mathbb{R}^{3}$, for $t>0, x \in \mathbb{R}^{3}$, denote electron and magnetic fields respectively. Initial data is given as

$$
\left.[n, u, E, B]\right|_{t=0}=\left[n_{0}, u_{0}, E_{0}, B_{0}\right], \quad x \in \mathbb{R}^{3},
$$

Date: September 6, 2018.

2000 Mathematics Subject Classification. 35Q35, 35P20.

Key words and phrases. Compressible Euler-Maxwell equations, stationary solutions, asymptotic stability.

*Corresponding author. Email: cjzhu@mail.ccnu.edu.cn . 
with the compatible conditions

$$
\nabla \cdot E_{0}=n_{b}(x)-n_{0}, \quad \nabla \cdot B_{0}=0, \quad x \in \mathbb{R}^{3} .
$$

The pressure function $p(\cdot)$ of the flow depending only on the density satisfies the power law $p(n)=A n^{\gamma}$ with constants $A>0$ and the adiabatic exponent $\gamma>1$. Constant $\nu>0$ is the velocity relaxation frequency. In this paper, we set $A=1, \nu=1$ without loss of generality. $n_{b}(x)$ denotes the stationary background ion density satisfying

$$
n_{b}(x) \rightarrow n_{\infty}, \quad \text { as } \quad|x| \rightarrow \infty,
$$

for a positive constant state $n_{\infty}>0$. Throughout this paper, we take $n_{\infty}=1$ for simplicity.

In comparison with the Euler-Maxwell system studied in [5], where the background density is a uniform constant, the naturally existing steady states of system (1.1) are no longer constants $[1,0,0,0]$. The stationary equations to the Cauchy problem (1.1)-(1.2) are given as

$$
\left\{\begin{array}{l}
\frac{1}{n_{s t}} \nabla p\left(n_{s t}\right)=-E_{s t}, \\
\nabla \times E_{s t}=0, \\
\nabla \cdot E_{s t}=n_{b}(x)-n_{s t} .
\end{array}\right.
$$

First, in this paper, we prove the existence of the stationary solutions to the Cauchy problem (1.1)-(1.2) under some conditions on the background density $n_{b}(x)$. For this purpose, let us define the weighted norm $\|\cdot\|_{W_{k}^{m, 2}}$ by

$$
\|g\|_{W_{k}^{m, 2}}=\left(\sum_{|\alpha| \leq m} \int_{\mathbb{R}^{3}}(1+|x|)^{k}\left|\partial_{x}^{\alpha} g(x)\right|^{2} d x\right)^{\frac{1}{2}},
$$

for suitable $g=g(x)$ and integers $m \geq 0, k \geq 0$.

Actually, one has the following theorem.

Theorem 1.1. For integers $m \geq 2$ and $k \geq 0$, suppose that $\left\|n_{b}-1\right\|_{W_{k}^{m, 2}}$ is small enough. Then the stationary problem (1.4) admits a unique solution $\left(n_{s t}, E_{s t}\right) \in L^{\infty}\left(0, T ; W_{k}^{m, 2}\right)$ satisfying

$$
\left\|n_{s t}-1\right\|_{W_{k}^{m, 2}} \leq C\left\|n_{b}-1\right\|_{W_{k}^{m, 2}}, \quad\left\|E_{s t}\right\|_{W_{k}^{m-1,2}} \leq C\left\|n_{b}-1\right\|_{W_{k}^{m, 2}}
$$

for some constant $C$.

There have been extensive investigations into the simplified Euler-Maxwell system where all the physical parameters are set to unity. For the one-fluid Euler-Maxwell system, by using the fractional Godunov scheme as well as the compensated compactness argument, Chen-Jerome-Wang in [3] proved global existence of weak solutions to the initial-boundary value problem in one space dimension for arbitrarily large initial data in $L^{\infty}$. Jerome in [15] established a local smooth solution theory for the Cauchy problem over $\mathbb{R}^{3}$ by adapting the classical semigroup-resolvent approach of Kato in [16]. Recently, Duan in [5] proved the existence and uniqueness of global solutions in the framework of smooth solutions with small amplitude, moreover the detailed analysis of Green's function to the linearized system was made to derive the optimal time-decay rates of perturbed solutions. The similar results are independently given by Ueda-Wang-Kawashima in [26] and Ueda-Kawashima in [25] by using the pure time-weighted energy method. For the the original two-fluid Euler-Maxwell systems with various parameters, the limits as some parameters go to zero have been studied recently. Peng-Wang in [19, 20, 21] justified the convergence of the one-fluid compressible Euler-Maxwell system to the incompressible Euler system, compressible Euler-Poisson system and an electron magnetohydrodynamics system for well-prepared smooth initial data. These asymptotic limits are respectively called non-relativistic limit, the quasi-neutral limit and the 
limit of their combination. Recently, Hajjej and Peng in [14 considered the zero-relaxation limits for periodic smooth solutions of Euler-Maxwell systems. For the 2-fluid Euler-Maxwell system, depending on the choice of physical parameters, especially the coefficients of $u_{ \pm}$were assumed $\nu_{+}=\nu_{-}$, Duan-Liu-Zhu in [7] obtained the existence and the time-decay rates of the solutions. Much more studies have been made for the Euler-Poisson system when the magnetic field is absent; see [12, 13, 17, 4, 18, 2] and references therein for discussion and analysis of the different issues such as the existence of global smooth irrotational flow [12] for an electron fluid and [13] for the ion dynamics, large time behavior of solutions [17], stability of star solutions [4, 18] and finite time blow-up [2].

However, there are few results on the global existence of solutions to the Euler-Maxwell system when the non-moving ions provide a nonconstant background $n_{b}(x)$, whereas in many papers related to one-fluid Euler-Maxwell system $n_{b}=1$. In this paper, we prove that there exists a stationary solution when the background density is a small perturbation of a positive constant state and we show the asymptotic stability of the stationary solution and then obtain the convergence rate of the global solution towards the stationary solution. The main result is stated as follows. Notations will be explained at the end of this section.

Theorem 1.2. Let $N \geq 3$ and (1.3) hold. Suppose $\left\|n_{b}-1\right\|_{W_{0}^{N+1,2}}$ is small enough. Then there are $\delta_{0}>0, C_{0}>0$ such that if

$$
\left\|\left[n_{0}-n_{s t}, u_{0}, E_{0}-E_{s t}, B_{0}\right]\right\|_{N} \leq \delta_{0},
$$

then, the Cauchy problem (1.1)-(1.2) admits a unique global solution $[n(t, x), u(t, x), E(t, x), B(t, x)]$ satisfying

$$
\left[n-n_{s t}, u, E-E_{s t}, B\right] \in C\left([0, \infty) ; H^{N}\left(\mathbb{R}^{3}\right)\right) \cap \operatorname{Lip}\left([0, \infty) ; H^{N-1}\left(\mathbb{R}^{3}\right)\right),
$$

and

$$
\sup _{t \geq 0}\left\|\left[n(t)-n_{s t}, u(t), E(t)-E_{s t}, B(t)\right]\right\|_{N} \leq C_{0}\left\|\left[n_{0}-n_{s t}, u_{0}, E_{0}-E_{s t}, B_{0}\right]\right\|_{N} .
$$

Moreover, there are $\delta_{1}>0, C_{1}>0$ such that if

$$
\left\|\left[n_{0}-n_{s t}, u_{0}, E_{0}-E_{s t}, B_{0}\right]\right\|_{N+3}+\left\|\left[u_{0}, E_{0}-E_{s t}, B_{0}\right]\right\|_{L^{1}} \leq \delta_{1},
$$

and $\left\|n_{b}-1\right\|_{W_{0}^{N+4,2}}$ is small enough, then the solution $[n(t, x), u(t, x), E(t, x), B(t, x)]$ satisfies that for any $t \geq 0$,

$$
\begin{gathered}
\left\|\left[n(t)-n_{s t}, u(t), B(t), E(t)-E_{s t}\right]\right\|_{N} \leq C_{1}(1+t)^{-\frac{3}{4}} \\
\left\|\nabla\left[n(t)-n_{s t}, u(t), B(t), E(t)-E_{s t}\right]\right\|_{N-1} \leq C_{1}(1+t)^{-\frac{5}{4}} .
\end{gathered}
$$

More precisely, if

$$
\left\|\left[n_{0}-n_{s t}, u_{0}, E_{0}-E_{s t}, B_{0}\right]\right\|_{6}+\left\|\left[u_{0}, E_{0}-E_{s t}, B_{0}\right]\right\|_{L^{1}} \leq \delta_{1},
$$

and $\left\|n_{b}-1\right\|_{W_{0}^{7,2}}$ is small enough, we have

$$
\begin{gathered}
\left\|\left[n(t)-n_{s t}, u(t)\right]\right\| \leq C_{1}(1+t)^{-\frac{5}{4}} \\
\left\|\left[E(t)-E_{s t}, B(t)\right]\right\| \leq C_{1}(1+t)^{-\frac{3}{4}}
\end{gathered}
$$

If

$$
\left\|\left[n_{0}-n_{s t}, u_{0}, E_{0}-E_{s t}, B_{0}\right]\right\|_{7}+\left\|\left[u_{0}, E_{0}-E_{s t}, B_{0}\right]\right\|_{L^{1}} \leq \delta_{1},
$$

and $\left\|n_{b}-1\right\|_{W_{0}^{8,2}}$ is small enough, then $E(t)$ satisfies

$$
\left\|E(t)-E_{s t}\right\| \leq C_{1}(1+t)^{-\frac{5}{4}} .
$$


The proof of existence in Theorem 1.2 is based on the classical energy method. As in [5], the key point is to obtain the uniform-in-time a priori estimates in the form of

$$
\mathcal{E}_{N}(\bar{V}(t))+\lambda \int_{0}^{t} \mathcal{D}_{N}(\bar{V}(s)) d s \leq \mathcal{E}_{N}\left(\bar{V}_{0}\right)
$$

where $\bar{V}(t)$ is the perturbation of solutions, and $\mathcal{E}_{N}(\cdot), \mathcal{D}_{N}(\cdot)$ denote the energy functional and energy dissipation rate functional. Here if we make the energy estimates like what Duan did in [5], it is difficult to control the highest-order derivative of $\bar{E}$ because of the regularityloss type in the sense that $[\bar{E}, \bar{B}]$ is time-space integrable up to $N-1$ order only. In this paper, we modify the energy estimates by choosing a weighted function $1+\sigma_{s t}+\Phi\left(\sigma_{s t}\right)$ which plays a vital role in closing the energy estimates.

Furthermore, for the convergence rates of perturbed solutions in Theorem 1.1, we can not analyze the corresponding linearized system of (1.1) around the steady state $\left[n_{s t}, 0, E_{s t}, 0\right]$ directly. In this case, the Fourier analysis fails due to the difficulty of variant coefficients. Here, the main idea follows from [5] for combining energy estimates with the linearized results in 5 . In the process of obtaining the fastest decay rates of the perturbed solution, the great difficulty is to deal with these linear nonhomogeneous sources including $\rho_{s t}$, which can not bring enough decay rates. Whereas in [5], the nonhomogeneous sources are at least quadratically nonlinear. To overcome this difficulty, we make iteration for the inequalities (4.11) and (4.12) together. In theorem 1.2, we only capture the same time-decay properties of $u, E-E_{s t}$ and $B$ as [5] except $n-n_{s t} .\left\|n-n_{s t}\right\|$ decays as $(1+t)^{-\frac{5}{4}}$ in the fastest way, because the nonhomogeneous sources containing $\rho_{s t}$ decay at most the same as $\sqrt{\mathcal{E}_{N}^{h}(\cdot)}$.

The similar work was done for Vlasov-Poisson-Boltzmann system, where the background density is also a function of spatial variable. Duan and Yang in [11] considered the stability of the stationary states which were given by an elliptic equation with the exponential nonlinearity. The optimal time-decay of the Vlasov-Poisson-Boltzmann system in $\mathbb{R}^{3}$ was obtained by Duan and Strain in [8]. We also mention the work Duan-Ukai-Yang-Zhao in [10], Duan-LiuUkai-Yang in [6] for the study of optimal convergence rates of the compressible Navier-Stokes equations with potential forces. Their proofs were based on the combination of spectral analysis and energy estimates. Recently, Duan-Ukai-Yang in [9] developed a method of the combination of the spectral analysis and energy estimates to deal with the optimal time decay for study of equations of gas motion.

We further remark the result in [11, the existence of solution to the elliptic equation $\Delta \phi=e^{\phi}-\bar{\rho}(x)$ has been proved when $\|\bar{\rho}-1\|_{W_{k}^{m, \infty}}$ is sufficiently small, where the weighted norm $\|\cdot\|_{W_{k}^{m}, \infty}$ is defined by

$$
\|g\|_{W_{k}^{m, \infty}}=\sup _{x \in \mathbb{R}^{3}}(1+|x|)^{k} \sum_{|\alpha| \leq m}\left|\partial_{x}^{\alpha} g(x)\right|
$$

for suitable $g=g(x)$ and integers $m \geq 0, k \geq 0$, the stability of the perturbed solutions can be proved when $\|\bar{\rho}-1\|_{W_{2}^{N+1, \infty}}$ is sufficiently small. We can also prove the stability of stationary solutions in the framework of [11] if $\left\|n_{b}-1\right\|_{W_{0}^{N+1, \infty}}$ is sufficiently small. In order to obtain the same convergence rates, $\left\|n_{b}-1\right\|_{W_{2}^{N+4, \infty}}$ should be sufficiently small in the process of dealing with $\rho_{s t} \bar{u}$ as in Section 4 ,

$$
\left\|\rho_{s t} \bar{u}\right\|_{L^{1}} \leq\left\|\rho_{s t}\right\|\|\bar{u}\| \leq C\left\|\rho_{s t}\right\|_{W_{2}^{N+4, \infty}}\|\bar{u}\| .
$$

Notice that $W_{2}^{N+4, \infty} \subseteq W_{0}^{N+4,2}$, it seems to be better to consider the existence of steady states in the weighted Sobolev space $W_{k}^{m, 2}$.

Let us introduce some notations for the use throughout this paper. $C$ denotes some positive (generally large) constant and $\lambda$ denotes some positive (generally small) constant, where both $C$ and $\lambda$ may take different values in different places. For two quantities $a$ and $b, a \sim b$ means 
$\lambda a \leq b \leq \frac{1}{\lambda} a$ for a generic constant $0<\lambda<1$. For any integer $m \geq 0$, we use $H^{m}, \dot{H}^{m}$ to denote the usual Sobolev space $H^{m}\left(\mathbb{R}^{3}\right)$ and the corresponding $m$-order homogeneous Sobolev space, respectively. Set $L^{2}=H^{m}$ when $m=0$. For simplicity, the norm of $H^{m}$ is denoted by $\|\cdot\|_{m}$ with $\|\cdot\|=\|\cdot\|_{0}$. We use $\langle\cdot, \cdot\rangle$ to denote the inner product over the Hilbert space $L^{2}\left(\mathbb{R}^{3}\right)$, i.e.

$$
\langle f, g\rangle=\int_{\mathbb{R}^{3}} f(x) g(x) d x, \quad f=f(x), \quad g=g(x) \in L^{2}\left(\mathbb{R}^{3}\right) .
$$

For a multi-index $\alpha=\left[\alpha_{1}, \alpha_{2}, \alpha_{3}\right]$, we denote $\partial^{\alpha}=\partial_{x_{1}}^{\alpha_{1}} \partial_{x_{2}}^{\alpha_{2}} \partial_{x_{3}}^{\alpha_{3}}$. The length of $\alpha$ is $|\alpha|=$ $\alpha_{1}+\alpha_{2}+\alpha_{3}$. For simplicity, we also set $\partial_{j}=\partial_{x_{j}}$ for $j=1,2,3$.

We conclude this section by stating the arrangement of the rest of this paper. In Section 2 , we prove the existence of the stationary solution. In Section 3, we reformulate the Cauchy problem under consideration and obtain asymptotic stability of solutions near the stationary state provided that the initial perturbation is sufficiently small. In Section 4, we study the time-decay rates of solutions to the stationary solutions by combining the $L^{p}$ - $L^{q}$ time-decay property of the linearized homogeneous system with time-weighted estimate.

\section{EXISTENCE OF STATIONARY SOLUTION}

In this section, we will prove the existence of stationary solutions to (1.4) by using the contraction mapping theorem. From $\left((1.4)_{2}\right.$, there exists $\phi_{s t}$ such that $E_{s t}=\nabla \phi_{s t}$, it turns equation (1.4) into

$$
\left\{\begin{array}{l}
\frac{1}{n_{s t}} \nabla p\left(n_{s t}\right)=-\nabla \phi_{s t}, \\
\Delta \phi_{s t}=n_{b}(x)-n_{s t} .
\end{array}\right.
$$

We introduce the nonlinear transformation (cf. [4])

$$
Q_{s t}=\frac{\gamma}{\gamma-1}\left(n_{s t}^{\gamma-1}-1\right) \text {. }
$$

From (2.1) and (2.2), we derive the following elliptic equation

$$
\Delta Q_{s t}=\left(\frac{\gamma-1}{\gamma} Q_{s t}+1\right)^{\frac{1}{\gamma-1}}-n_{b}(x) .
$$

For convenience, we replace $Q_{s t}$ by $\phi$ in the following. Equation (2.3) can be rewritten as the integral form

$$
\phi=T(\phi)=G *\left(\left(\frac{\gamma-1}{\gamma} \phi+1\right)^{\frac{1}{\gamma-1}}-\frac{1}{\gamma} \phi-n_{b}(x)\right),
$$

where $G=G(x)$ given by

$$
G(x)=-\frac{1}{4 \pi|x|} e^{-\frac{1}{\sqrt{\gamma}}|x|}
$$

is the fundamental solution to the linear elliptic equation $\Delta_{x} G-\frac{1}{\gamma} G=0$. Thus (2.3) admits a solution if and only if the nonlinear mapping $T$ has a fixed point. Define

$$
\mathscr{B}_{m, k}(B)=\left\{\phi \in W_{k}^{m, 2}\left(\mathbb{R}^{3}\right) ;\|\phi\|_{W_{k}^{m, 2}} \leq B\left\|n_{b}-1\right\|_{W_{k}^{m, 2}}, m \geq 2\right\}
$$

for some constant $B$ to be determined later. Next, we prove that if $\left\|n_{b}-1\right\|_{W_{k}^{m, 2}}$ is small enough, there exists a constant $B$ such that $T: \mathscr{B}_{m, k}(B) \rightarrow \mathscr{B}_{m, k}(B)$ is a contraction 
mapping. In fact, for simplicity, let us denote

$$
g(x)=\left(\frac{\gamma-1}{\gamma} x+1\right)^{\frac{1}{\gamma-1}}-\frac{1}{\gamma} x-1 .
$$

Then it holds that

$$
T(\phi)(x)=-\int_{\mathbb{R}^{3}} \frac{1}{4 \pi|x-y|} e^{-\frac{1}{\sqrt{\gamma}}|x-y|}\left[g(\phi(y))-\left(n_{b}(y)-1\right)\right] d y .
$$

Taking derivatives $\partial_{x}^{\alpha}$ on both sides of (2.4), one has

$$
\begin{aligned}
\partial_{x}^{\alpha} T(\phi)(x) & =-(-1)^{|\alpha|} \int_{\mathbb{R}^{3}} \frac{1}{4 \pi|x-y|} e^{-\frac{1}{\sqrt{\gamma}}|x-y|}\left[\partial_{y}^{\alpha} g(\phi(y))-\partial_{y}^{\alpha}\left(n_{b}(y)-1\right)\right] d y \\
& =-(-1)^{|\alpha|} G *\left(\partial^{\alpha} g(\phi)-\partial^{\alpha}\left(n_{b}-1\right)\right) .
\end{aligned}
$$

Here let's list some properties of the operator $G *$.

Lemma 2.1. For any $k \geq 0$, it holds that

$$
\int_{\mathbb{R}^{3}} \frac{1}{|y|} e^{-\frac{1}{\sqrt{\gamma}}|y|} \frac{1}{(1+|x-y|)^{k}} d y \leq \frac{C_{k}}{(1+|x|)^{k}},
$$

and for any $f \in W_{k}^{m, 2}$,

$$
\left\|(1+|x|)^{\frac{k}{2}}(G * f)\right\| \leq C_{k}^{\frac{1}{2}}\|G\|_{L^{1}}^{\frac{1}{2}}\left\|(1+|x|)^{\frac{k}{2}} f\right\| .
$$

Proof. (2.6) has been proved in [11]. We only prove (2.7) by using (2.6).

$$
\begin{aligned}
\left|\int_{\mathbb{R}^{3}} G(x-y) f(y) d y\right| & \leq \int_{\mathbb{R}^{3}} \frac{|G(x-y)|^{\frac{1}{2}}}{(1+|y|)^{\frac{k}{2}}}|G(x-y)|^{\frac{1}{2}}(1+|y|)^{\frac{k}{2}}|f(y)| d y \\
& \leq\left(\int_{\mathbb{R}^{3}} \frac{|G(x-y)|}{(1+|y|)^{k}} d y\right)^{\frac{1}{2}}\left(\int_{\mathbb{R}^{3}}|G(x-y)|(1+|y|)^{k}|f(y)|^{2} d y\right)^{\frac{1}{2}} \\
& \leq \frac{C_{k}^{\frac{1}{2}}}{(1+|x|)^{\frac{k}{2}}}\left(\int_{\mathbb{R}^{3}}|G(x-y)|(1+|y|)^{k}|f(y)|^{2} d y\right)^{\frac{1}{2}} .
\end{aligned}
$$

Then

$$
\begin{aligned}
& \int_{\mathbb{R}^{3}}(1+|x|)^{k}\left|\int_{\mathbb{R}^{3}} G(x-y) f(y) d y\right|^{2} d x \\
\leq & C_{k} \int_{\mathbb{R}^{3}} \int_{\mathbb{R}^{3}}|G(x-y)|(1+|y|)^{k}|f(y)|^{2} d y d x \\
= & C_{k} \int_{\mathbb{R}^{3}} \int_{\mathbb{R}^{3}}|G(x-y)|(1+|y|)^{k}|f(y)|^{2} d x d y \\
= & C_{k} \int_{\mathbb{R}^{3}}(1+|y|)^{k}|f(y)|^{2} d y \int_{\mathbb{R}^{3}}|G(x-y)| d x \\
= & C_{k}\|G\|_{L^{1}}\left\|(1+|x|)^{\frac{k}{2}} f\right\|^{2} .
\end{aligned}
$$

Remark: When $k=0, C_{k}=\|G\|_{L^{1}}$, (2.7) is in accordance with Young inequality.

By (2.7) and (2.5), one has

$$
\begin{aligned}
& \left\|(1+|x|)^{\frac{k}{2}} \partial_{x}^{\alpha} T(\phi)(x)\right\| \\
\leq & \left.C\left\|(1+|x|)^{\frac{k}{2}} \partial^{\alpha} g(\phi)\right\|+C \|(1+|x|)^{\frac{k}{2}} \partial^{\alpha}\left(n_{b}-1\right)\right) \| .
\end{aligned}
$$


By the definition (1.5) of the norm $\|\cdot\|_{W_{k}^{m, 2}}$, one has

$$
\begin{aligned}
\|T(\phi)(x)\|_{W_{k}^{m, 2}} & =\left(\sum_{|\alpha| \leq m}\left\|(1+|x|)^{\frac{k}{2}} \partial_{x}^{\alpha} T(\phi)(x)\right\|^{2}\right)^{\frac{1}{2}} \\
& \leq C\left(\sum_{|\alpha| \leq m}\left\|(1+|x|)^{\frac{k}{2}} \partial^{\alpha} g(\phi)\right\|^{2}\right)^{\frac{1}{2}}+C\left(\sum_{|\alpha| \leq m}\left\|(1+|x|)^{\frac{k}{2}} \partial^{\alpha}\left(n_{b}-1\right)\right\|^{2}\right)^{\frac{1}{2}} \\
(2.8) & \leq C\left(\sum_{|\alpha| \leq m}\left\|(1+|x|)^{\frac{k}{2}} \partial^{\alpha} g(\phi)\right\|^{2}\right)^{\frac{1}{2}}+C\left\|n_{b}-1\right\|_{W_{k}^{m, 2} .}
\end{aligned}
$$

$$
g(\phi)=\int_{0}^{1} \int_{0}^{\theta} g^{\prime \prime}(\tau \phi) d \tau d \theta \phi^{2} \triangleq h(\phi) \phi^{2}
$$

where $g^{\prime \prime}(x)=\frac{2-\gamma}{\gamma^{2}}\left(\frac{\gamma-1}{\gamma} x+1\right)^{\frac{3-2 \gamma}{\gamma-1}}$.

It is straightforward to check that

$$
\left\|(1+|x|)^{\frac{k}{2}} \partial^{\alpha}\left(h(\phi) \phi^{2}\right)\right\| \leq \sum_{\beta_{1}+\beta_{2}+\beta_{3}=\alpha} C_{\beta_{1}, \beta_{2}, \beta_{3}}^{\alpha}\left\|(1+|x|)^{\frac{k}{2}} \partial^{\beta_{1}} h(\phi) \partial^{\beta_{2}} \phi \partial^{\beta_{3}} \phi\right\| .
$$

In addition, one has the following claim.

Claim:

$$
\left\|(1+|x|)^{\frac{k}{2}} \partial^{\beta_{1}} h(\phi) \partial^{\beta_{2}} \phi \partial^{\beta_{3}} \phi\right\| \leq C\|\phi\|_{W_{k}^{m, 2}}^{2} .
$$

Proof of claim: We prove (2.9) by two cases.

Case 1. $\beta_{1}=0$. In this case, $\left|\beta_{2}\right|+\left|\beta_{3}\right| \leq m$, thus one can suppose $\left|\beta_{2}\right| \leq\left[\frac{m}{2}\right]$ by the symmetry of $\beta_{2}$ and $\beta_{3}$. This deduces

$$
\begin{aligned}
\left\|(1+|x|)^{\frac{k}{2}} h(\phi) \partial^{\beta_{2}} \phi \partial^{\beta_{3}} \phi\right\| & \leq\|h(\phi)\|_{L^{\infty}}\left\|\partial^{\beta_{2}} \phi\right\|_{L^{6}}\left\|(1+|x|)^{\frac{k}{2}} \partial^{\beta_{3}} \phi\right\|_{L^{3}} \\
& \leq C\left\|\nabla \partial^{\beta_{2}} \phi\right\|\left\|(1+|x|)^{\frac{k}{2}} \partial^{\beta_{3}} \phi\right\|_{1} \\
& \leq C\|\phi\|_{W_{k}^{m, 2}}^{2} .
\end{aligned}
$$

Here, we have used that $h(\cdot)$ is a continuous function in the argument,

$$
\|\phi\|_{L^{\infty}} \leq C\|\nabla \phi\|_{H^{1}} \leq C\|\phi\|_{W_{k}^{m, 2}} \leq C\left\|n_{b}-1\right\|_{W_{k}^{m, 2}} \ll 1,
$$

and $m \geq 2$.

Case 2. $\left|\beta_{1}\right| \geq 1$. Notice that

$$
\partial^{\beta_{1}} h(\phi)=\sum_{l=1}^{\left|\beta_{1}\right|} h^{(l)}(\phi) \sum_{\gamma_{1}+\gamma_{2}+\cdots \gamma_{l}=\beta_{1}} C_{\gamma_{1}, \gamma_{2}, \cdots \gamma_{l}} \Pi_{i=1}^{l} \partial^{\gamma_{i}} \phi,
$$

(2.9) can be similarly obtained because $h^{(m)}(\phi)$ is also bounded.

Putting (2.9) into (2.8), and using the above estimates, one has

$$
\|T(\phi)(x)\|_{W_{k}^{m, 2}} \leq C B^{2}\left\|n_{b}-1\right\|_{W_{k}^{m, 2}}^{2}+C\left\|n_{b}-1\right\|_{W_{k}^{m, 2}} .
$$

Finally, for any $\phi_{1}=\phi_{1}(x)$ and $\phi_{2}=\phi_{2}(x)$, it holds that

$$
T\left(\phi_{1}\right)-T\left(\phi_{2}\right)=G *\left(g\left(\phi_{1}\right)-g\left(\phi_{2}\right)\right)
$$


with

$$
g\left(\phi_{1}\right)-g\left(\phi_{2}\right)=\int_{0}^{1} g^{\prime}\left(\theta \phi_{1}+(1-\theta) \phi_{2}\right) d \theta\left(\phi_{1}-\phi_{2}\right)
$$

Notice that for any $\phi=\phi(x)$,

$$
\begin{aligned}
g^{\prime}(\phi) & =\frac{1}{\gamma}\left(\frac{\gamma-1}{\gamma} \phi+1\right)^{\frac{2-\gamma}{\gamma-1}}-\frac{1}{\gamma} \\
& =\int_{0}^{1} \frac{2-\gamma}{\gamma^{2}}\left(\frac{\gamma-1}{\gamma} \theta \phi+1\right)^{\frac{3-2 \gamma}{\gamma-1}} d \theta \phi
\end{aligned}
$$

Then the same computations as for (2.10) yield

$$
\begin{aligned}
& \left\|T\left(\phi_{1}\right)-T\left(\phi_{2}\right)\right\|_{W_{k}^{m, 2}} \\
\leq & C\left(\left\|\phi_{1}\right\|_{W_{k}^{m, 2}}+\left\|\phi_{2}\right\|_{W_{k}^{m, 2}}\right)\left\|\phi_{1}-\phi_{2}\right\|_{W_{k}^{m, 2}} .
\end{aligned}
$$

Combining (2.10) with (2.11), the standard argument implies that $T$ has a unique fixed point $\phi$ in $\mathscr{B}_{m, k}(B)$ for a proper constant $B$ provided that $\left\|n_{b}-1\right\|_{W_{k}^{m, 2}}$ is small enough. This completes Theorem 1.1.

Let us conclude this section with a remark. The existence of solutions to the elliptic equation (2.3) can also be proved in the framework of [11] when $\left\|n_{b}-1\right\|_{W_{k}^{m, \infty}}$ is sufficiently small. We consider the existence when $\left\|n_{b}-1\right\|_{W_{k}^{m, 2}}$ is sufficiently small in order to derive the more general conclusion. In fact, in the process of dealing with the stability and convergence rates, only the smallness of $\left\|n_{b}-1\right\|_{W_{0}^{m, 2}}$ is assumed, and the space decay at infinity of $n_{b}(x)-1$ is not needed.

\section{Stability of Stationary SOLUtion}

3.1. Reformulation of the problem. Let $[n, u, E, B]$ be a smooth solution to the Cauchy problem of the Euler-Maxwell system (1.1) with given initial data (1.2) satisfying (1.3). Set

$$
\left\{\begin{array}{l}
\sigma(t, x)=\frac{2}{\gamma-1}\left\{\left[n\left(\frac{t}{\sqrt{\gamma}}, x\right)\right]^{\frac{\gamma-1}{2}}-1\right\}, \quad v=\frac{1}{\sqrt{\gamma}} u\left(\frac{t}{\sqrt{\gamma}}, x\right), \\
\tilde{E}=\frac{1}{\sqrt{\gamma}} E\left(\frac{t}{\sqrt{\gamma}}, x\right), \quad \tilde{B}=\frac{1}{\sqrt{\gamma}} B\left(\frac{t}{\sqrt{\gamma}}, x\right) .
\end{array}\right.
$$

Then, $V:=[\sigma, v, \tilde{E}, \tilde{B}]$ satisfies 


$$
\left\{\begin{array}{l}
\partial_{t} \sigma+\left(\frac{\gamma-1}{2} \sigma+1\right) \nabla \cdot v+v \cdot \nabla \sigma=0, \\
\partial_{t} v+v \cdot \nabla v+\left(\frac{\gamma-1}{2} \sigma+1\right) \nabla \sigma=-\left(\frac{1}{\sqrt{\gamma}} \tilde{E}+v \times \tilde{B}\right)-\frac{1}{\sqrt{\gamma}} v, \\
\partial_{t} \tilde{E}-\frac{1}{\sqrt{\gamma}} \nabla \times \tilde{B}=\frac{1}{\sqrt{\gamma}} v+\frac{1}{\sqrt{\gamma}}[\Phi(\sigma)+\sigma] v, \\
\partial_{t} \tilde{B}+\frac{1}{\sqrt{\gamma}} \nabla \times \tilde{E}=0, \\
\nabla \cdot \tilde{E}=-\frac{1}{\sqrt{\gamma}}[\Phi(\sigma)+\sigma]+\frac{1}{\sqrt{\gamma}}\left(n_{b}(x)-1\right), \quad \nabla \cdot \tilde{B}=0, \quad t>0, x \in \mathbb{R}^{3},
\end{array}\right.
$$

with initial data

$$
\left.V\right|_{t=0}=V_{0}:=\left[\sigma_{0}, v_{0}, \tilde{E}_{0}, \tilde{B}_{0}\right], \quad x \in \mathbb{R}^{3} .
$$

Here, $\Phi(\cdot)$ is defined by

$$
\Phi(\sigma)=\left(\frac{\gamma-1}{2} \sigma+1\right)^{\frac{2}{\gamma-1}}-\sigma-1
$$

and $V_{0}=\left[\sigma_{0}, v_{0}, \tilde{E}_{0}, \tilde{B}_{0}\right]$ is given from $\left[n_{0}, u_{0}, E_{0}, B_{0}\right]$ according to the transform (3.1), and hence $V_{0}$ satisfies

$$
\nabla \cdot \tilde{E}_{0}=-\frac{1}{\sqrt{\gamma}}\left[\Phi\left(\sigma_{0}\right)+\sigma_{0}\right]+\frac{1}{\sqrt{\gamma}}\left(n_{b}(x)-1\right), \quad \nabla \cdot \tilde{B}_{0}=0, \quad x \in \mathbb{R}^{3} .
$$

On the other hand, set

$$
\sigma_{s t}(x)=\frac{2}{\gamma-1}\left\{n_{s t}(x)^{\frac{\gamma-1}{2}}-1\right\}, \quad \tilde{E}_{s t}=\frac{1}{\sqrt{\gamma}} E_{s t}(x)
$$

Then, $\left[\sigma_{s t}, \tilde{E}_{s t}\right]$ satisfies

$$
\left\{\begin{array}{l}
\left(\frac{\gamma-1}{2} \sigma_{s t}+1\right) \nabla \sigma_{s t}=-\frac{1}{\sqrt{\gamma}} \tilde{E}_{s t}, \\
\frac{1}{\sqrt{\gamma}} \nabla \times \tilde{E}_{s t}=0 \\
\nabla \cdot \tilde{E}_{s t}=\frac{1}{\sqrt{\gamma}}\left(n_{b}(x)-1\right)-\frac{1}{\sqrt{\gamma}}\left(\Phi\left(\sigma_{s t}\right)+\sigma_{s t}\right) .
\end{array}\right.
$$

Based on the existence result proved in Section 2, we will study the stability of the stationary state $\left[\sigma_{s t}, 0, \tilde{E}_{s t}, 0\right]$. Set the perturbations $[\bar{\sigma}, \bar{v}, \bar{E}, \bar{B}]$ by

$$
\bar{\sigma}=\sigma-\sigma_{s t}, \quad \bar{v}=v, \quad \bar{E}=\tilde{E}-\tilde{E}_{s t}, \quad \bar{B}=\tilde{B} .
$$

Combining (3.2) with (3.7), then $\bar{V}:=[\bar{\sigma}, \bar{v}, \bar{E}, \bar{B}]$ satisfies 


$$
\left\{\begin{array}{l}
\partial_{t} \bar{\sigma}+\left(\frac{\gamma-1}{2} \bar{\sigma}+1\right) \nabla \cdot \bar{v}+\bar{v} \cdot \nabla \bar{\sigma}+\bar{v} \cdot \nabla \sigma_{s t}+\frac{\gamma-1}{2} \sigma_{s t} \nabla \cdot \bar{v}=0, \\
\partial_{t} \bar{v}+\bar{v} \cdot \nabla \bar{v}+\left(\frac{\gamma-1}{2} \bar{\sigma}+1\right) \nabla \bar{\sigma}+\frac{\gamma-1}{2} \bar{\sigma} \nabla \sigma_{s t}+\frac{\gamma-1}{2} \sigma_{s t} \nabla \bar{\sigma}=-\left(\frac{1}{\sqrt{\gamma}} \bar{E}+\bar{v} \times \bar{B}\right)-\frac{1}{\sqrt{\gamma}} \bar{v} \\
\partial_{t} \bar{E}-\frac{1}{\sqrt{\gamma}} \nabla \times \bar{B}=\frac{1}{\sqrt{\gamma}} \bar{v}+\frac{1}{\sqrt{\gamma}}\left[\Phi\left(\bar{\sigma}+\sigma_{s t}\right)+\bar{\sigma}+\sigma_{s t}\right] \bar{v}, \\
\partial_{t} \bar{B}+\frac{1}{\sqrt{\gamma}} \nabla \times \bar{E}=0, \\
\nabla \cdot \bar{E}=-\frac{1}{\sqrt{\gamma}}\left[\Phi\left(\bar{\sigma}+\sigma_{s t}\right)-\Phi\left(\sigma_{s t}\right)\right]-\frac{1}{\sqrt{\gamma}} \bar{\sigma}, \quad \nabla \cdot \bar{B}=0, \quad t>0, x \in \mathbb{R}^{3},
\end{array}\right.
$$

with initial data

$$
\left.\bar{V}\right|_{t=0}=\bar{V}_{0}:=\left[\sigma_{0}-\sigma_{s t}, v_{0}, \tilde{E}_{0}-\tilde{E}_{s t}, \tilde{B}_{0}\right], \quad x \in \mathbb{R}^{3} .
$$

Here, $\Phi(\cdot)$ is defined by (3.4), and $\bar{V}_{0}$ satisfies

$$
\nabla \cdot \bar{E}_{0}=-\frac{1}{\sqrt{\gamma}}\left[\Phi\left(\bar{\sigma}_{0}+\sigma_{s t}\right)-\Phi\left(\sigma_{s t}\right)\right]-\frac{1}{\sqrt{\gamma}} \bar{\sigma}_{0}, \quad \nabla \cdot \bar{B}_{0}=0, \quad t>0, x \in \mathbb{R}^{3} .
$$

In what follows, we suppose the integer $N \geq 3$. Besides, for $\bar{V}=[\bar{\sigma}, \bar{v}, \bar{E}, \bar{B}]$, we define the full instant energy functional $\mathcal{E}_{N}(\bar{V}(t))$, the high-order instant energy functional $\mathcal{E}_{N}^{h}(\bar{V}(t))$, and the dissipation rates $\mathcal{D}_{N}(\bar{V}(t)), \mathcal{D}_{N}^{h}(\bar{V}(t))$ by

$$
\begin{aligned}
\mathcal{E}_{N}(\bar{V}(t))= & \sum_{|\alpha| \leq N} \int_{\mathbb{R}^{3}}\left(1+\sigma_{s t}+\Phi\left(\sigma_{s t}\right)\right)\left(\left|\partial^{\alpha} \bar{\sigma}\right|^{2}+\left|\partial^{\alpha} \bar{v}\right|^{2}\right) d x+\|[\bar{E}, \bar{B}]\|_{N}^{2} \\
& +\kappa_{1} \sum_{|\alpha| \leq N-1}\left\langle\partial^{\alpha} \bar{v}, \nabla \partial^{\alpha} \bar{\sigma}\right\rangle+\kappa_{2} \sum_{|\alpha| \leq N-1}\left\langle\partial^{\alpha} \bar{v}, \partial^{\alpha} \bar{E}\right\rangle \\
& -\kappa_{3} \sum_{|\alpha| \leq N-2}\left\langle\nabla \times \partial^{\alpha} \bar{E}, \partial^{\alpha} \bar{B}\right\rangle,
\end{aligned}
$$

and

$$
\begin{aligned}
\mathcal{E}_{N}^{h}(\bar{V}(t)) & =\sum_{1 \leq|\alpha| \leq N} \int_{\mathbb{R}^{3}}\left(1+\sigma_{s t}+\Phi\left(\sigma_{s t}\right)\right)\left(\left|\partial^{\alpha} \bar{\sigma}\right|^{2}+\left|\partial^{\alpha} \bar{v}\right|^{2}\right) d x+\|\nabla[\bar{E}, \bar{B}]\|_{N-1}^{2} \\
& +\kappa_{1} \sum_{1 \leq|\alpha| \leq N-1}\left\langle\partial^{\alpha} \bar{v}, \nabla \partial^{\alpha} \bar{\sigma}\right\rangle+\kappa_{2} \sum_{1 \leq|\alpha| \leq N-1}\left\langle\partial^{\alpha} \bar{v}, \partial^{\alpha} \bar{E}\right\rangle \\
& -\kappa_{3} \sum_{1 \leq|\alpha| \leq N-2}\left\langle\nabla \times \partial^{\alpha} \bar{E}, \partial^{\alpha} \bar{B}\right\rangle,
\end{aligned}
$$

respectively, where $0<\kappa_{3} \ll \kappa_{2} \ll \kappa_{1} \ll 1$ are constants to be properly chosen in the later proof. Notice that since all constants $\kappa_{i}(i=1,2,3)$ are small enough, one has

$$
\mathcal{E}_{N}(\bar{V}(t)) \sim\|[\bar{\sigma}, \bar{v}, \bar{E}, \bar{B}]\|_{N}^{2}, \quad \mathcal{E}_{N}^{h}(\bar{V}(t)) \sim\|\nabla[\bar{\sigma}, \bar{v}, \bar{E}, \bar{B}]\|_{N-1}^{2} .
$$

We further define the dissipation rates $\mathcal{D}_{N}(\bar{V}(t)), \mathcal{D}_{N}^{h}(\bar{V}(t))$ by

$$
\begin{aligned}
\mathcal{D}_{N}(\bar{V}(t))=\sum_{|\alpha| \leq N} \int_{\mathbb{R}^{3}}\left(1+\sigma_{s t}\right. & \left.+\Phi\left(\sigma_{s t}\right)\right)\left|\partial^{\alpha} \bar{v}\right|^{2} d x \\
& +\|\bar{\sigma}\|_{N}^{2}+\|\nabla[\bar{E}, \bar{B}]\|_{N-2}^{2}+\|\bar{E}\|^{2},
\end{aligned}
$$


and

$$
\begin{aligned}
\mathcal{D}_{N}^{h}(\bar{V}(t))=\sum_{1 \leq|\alpha| \leq N} \int_{\mathbb{R}^{3}}\left(1+\sigma_{s t}\right. & \left.+\Phi\left(\sigma_{s t}\right)\right)\left|\partial^{\alpha} \bar{v}\right|^{2} d x \\
& +\|\nabla \bar{\sigma}\|_{N-1}^{2}+\left\|\nabla^{2}[\bar{E}, \bar{B}]\right\|_{N-3}^{2}+\|\nabla \bar{E}\|^{2}
\end{aligned}
$$

Then, concerning the reformulated Cauchy problem (3.8)-(3.9), one has the following global existence result.

Proposition 3.1. Suppose that $\left\|n_{b}-1\right\|_{W_{0}^{N+1,2}}$ is small enough and (3.10) holds for given initial data $\bar{V}_{0}=\left[\sigma_{0}-\sigma_{s t}, v_{0}, \tilde{E}_{0}-\tilde{E}_{s t}, \tilde{B}_{0}\right]$. Then, there are $\mathcal{E}_{N}(\cdot)$ and $\mathcal{D}_{N}(\cdot)$ in the form (3.11) and (3.13) such that the following holds true:

If $\mathcal{E}_{N}\left(\bar{V}_{0}\right)>0$ is small enough, the Cauchy problem (3.8)-(3.9) admits a unique global nonzero solution $\bar{V}=\left[\sigma-\sigma_{\text {st }}, v, \tilde{E}-\tilde{E}_{\text {st }}, \tilde{B}\right]$ satisfying

$$
\bar{V} \in C\left([0, \infty) ; H^{N}\left(\mathbb{R}^{3}\right)\right) \cap \operatorname{Lip}\left([0, \infty) ; H^{N-1}\left(\mathbb{R}^{3}\right)\right),
$$

and

$$
\mathcal{E}_{N}(\bar{V}(t))+\lambda \int_{0}^{t} \mathcal{D}_{N}(\bar{V}(s)) d s \leq \mathcal{E}_{N}\left(\bar{V}_{0}\right)
$$

for any $t \geq 0$.

Moreover, solutions obtained in Proposition 3.1 indeed decay in time with some rates under some extra regularity and integrability conditions on initial data. For that, given $\bar{V}_{0}=\left[\sigma_{0}-\sigma_{s t}, v_{0}, \tilde{E}_{0}-\tilde{E}_{s t}, \tilde{B}_{0}\right]$, set $\epsilon_{m}\left(\bar{V}_{0}\right)$ as

$$
\epsilon_{m}\left(\bar{V}_{0}\right)=\left\|\bar{V}_{0}\right\|_{m}+\left\|\left[v_{0}, \tilde{E}_{0}-\tilde{E}_{s t}, \tilde{B}_{0}\right]\right\|_{L^{1}},
$$

for the integer $m \geq 6$. Then one has the following proposition.

Proposition 3.2. Suppose that $\left\|n_{b}-1\right\|_{W_{0}^{N+4,2}}$ is small enough and (3.10) holds for given initial data $\bar{V}_{0}=\left[\sigma_{0}-\sigma_{s t}, v_{0}, \tilde{E}_{0}-\tilde{E}_{s t}, \tilde{B}_{0}\right]$. If $\epsilon_{N+3}\left(\bar{V}_{0}\right)>0$ is small enough, then the solution $\bar{V}=\left[\sigma-\sigma_{s t}, v, \tilde{E}-\tilde{E}_{s t}, \tilde{B}\right]$ satisfies

$$
\|\bar{V}(t)\|_{N} \leq C \epsilon_{N+3}\left(\bar{V}_{0}\right)(1+t)^{-\frac{3}{4}},
$$

and

$$
\|\nabla \bar{V}(t)\|_{N-1} \leq C \epsilon_{N+3}\left(\bar{V}_{0}\right)(1+t)^{-\frac{5}{4}}
$$

for any $t \geq 0$.

3.2. a priori estimates. In this subsection, we prove that the stationary solution obtained in Section 2 is stable under small initial perturbation. We begin to use the refined energy method to obtain some uniform-in-time a priori estimates for smooth solutions to the Cauchy problem (3.8)-(3.9). To the end, let us denote

$$
\delta=\left\|\sigma_{s t}\right\|_{W_{0}^{N+1,2}}=\left(\sum_{|\alpha| \leq N+1} \int_{\mathbb{R}^{3}}\left|\partial_{x}^{\alpha} \sigma_{s t}\right|^{2} d x\right)^{\frac{1}{2}}
$$


for simplicity of presentation. A careful look at the proof of Theorem 1.1 shows that

$$
\begin{aligned}
\sigma_{s t} & =\frac{2}{\gamma-1}\left\{n_{s t}^{\frac{\gamma-1}{2}}-1\right\} \\
& =\frac{2}{\gamma-1}\left\{\left(\frac{\gamma-1}{\gamma} Q_{s t}+1\right)^{\frac{1}{2}}-1\right\} \\
& =\frac{2}{\gamma} \frac{Q_{s t}}{\left(\frac{\gamma-1}{\gamma} Q_{s t}+1\right)^{\frac{1}{2}}+1} \sim Q_{s t} .
\end{aligned}
$$

It follows that $\delta \leq C\left\|Q_{s t}\right\|_{W_{0}^{N+1,2}} \leq C\left\|n_{b}-1\right\|_{W_{0}^{N+1,2}}$ is small enough. Notice that (3.8) is a quasi-linear symmetric hyperbolic system. The main goal of this subsection is to prove

Theorem 3.1. (a priori estimates). Let $0<T \leq \infty$ be given. Suppose $\bar{V}:=[\bar{\sigma}, \bar{v}, \bar{E}, \bar{B}] \in$ $C\left([0, T) ; H^{N}\left(\mathbb{R}^{3}\right)\right)$ is smooth for $T>0$ with

$$
\sup _{0 \leq t<T}\|\bar{V}(t)\|_{N} \leq 1
$$

and assume that $\bar{V}$ solves the system (3.8) for $t \in(0, T)$. Then, there are $\mathcal{E}_{N}(\cdot)$ and $\mathcal{D}_{N}(\cdot)$ in the form (3.11) and (3.13) such that

$$
\frac{d}{d t} \mathcal{E}_{N}(\bar{V}(t))+\lambda \mathcal{D}_{N}(\bar{V}(t)) \leq C\left[\mathcal{E}_{N}(\bar{V}(t))^{\frac{1}{2}}+\mathcal{E}_{N}(\bar{V}(t))+\delta\right] \mathcal{D}_{N}(\bar{V}(t))
$$

for any $0 \leq t<T$.

Proof. The proof is divided into five steps.

Step 1. It holds that

$$
\begin{aligned}
& \quad \frac{1}{2} \frac{d}{d t}\left(\sum_{|\alpha| \leq N} \int_{\mathbb{R}^{3}}\left(1+\sigma_{s t}+\Phi\left(\sigma_{s t}\right)\right)\left(\left|\partial^{\alpha} \bar{\sigma}\right|^{2}+\left|\partial^{\alpha} \bar{v}\right|^{2}\right) d x+\|[\bar{E}, \bar{B}]\|_{N}^{2}\right) \\
& \quad+\frac{1}{\sqrt{\gamma}} \sum_{|\alpha| \leq N} \int_{\mathbb{R}^{3}}\left(1+\sigma_{s t}+\Phi\left(\sigma_{s t}\right)\right)\left|\partial^{\alpha} \bar{v}\right|^{2} d x \\
& \leq C\left(\|\bar{V}\|_{N}+\delta\right)\left(\|[\bar{\sigma}, \bar{v}]\|^{2}+\|\nabla[\bar{\sigma}, \bar{v}]\|_{N-1}^{2}+\|\nabla \bar{E}\|_{N-2}^{2}\right) .
\end{aligned}
$$

In fact, applying $\partial^{\alpha}$ to the first two equations of (3.8) for $|\alpha| \leq N$ and multiplying them by $\left(1+\sigma_{s t}+\Phi\left(\sigma_{s t}\right)\right) \partial^{\alpha} \bar{\sigma}$ and $\left(1+\sigma_{s t}+\Phi\left(\sigma_{s t}\right)\right) \partial^{\alpha} \bar{v}$ respectively, taking integrations in $x$ and then using integration by parts give

$$
\begin{aligned}
& \frac{1}{2} \frac{d}{d t} \int_{\mathbb{R}^{3}}\left(1+\sigma_{s t}+\Phi\left(\sigma_{s t}\right)\right)\left(\left|\partial^{\alpha} \bar{\sigma}\right|^{2}+\left|\partial^{\alpha} \bar{v}\right|^{2}\right) d x+\frac{1}{\sqrt{\gamma}}\left\langle\partial^{\alpha} \bar{E},\left(1+\sigma_{s t}+\Phi\left(\sigma_{s t}\right)\right) \partial^{\alpha} \bar{v}\right\rangle \\
& +\frac{1}{\sqrt{\gamma}} \int_{\mathbb{R}^{3}}\left(1+\sigma_{s t}+\Phi\left(\sigma_{s t}\right)\right)\left|\partial^{\alpha} \bar{v}\right|^{2} d x=-\sum_{\beta<\alpha} C_{\beta}^{\alpha} I_{\alpha, \beta}(t)+I_{1}(t) .
\end{aligned}
$$

Here, $I_{\alpha, \beta}(t)=I_{\alpha, \beta}^{(\sigma)}(t)+I_{\alpha, \beta}^{(v)}(t)$ with

$$
\begin{aligned}
I_{\alpha, \beta}^{(\sigma)}(t)= & \left\langle\partial^{\alpha-\beta} \bar{v} \cdot \nabla \partial^{\beta} \bar{\sigma},\left(1+\sigma_{s t}+\Phi\left(\sigma_{s t}\right)\right) \partial^{\alpha} \bar{\sigma}\right\rangle \\
& +\frac{\gamma-1}{2}\left\langle\partial^{\alpha-\beta} \bar{\sigma} \partial^{\beta} \nabla \cdot \bar{v},\left(1+\sigma_{s t}+\Phi\left(\sigma_{s t}\right)\right) \partial^{\alpha} \bar{\sigma}\right\rangle \\
& +\frac{\gamma-1}{2}\left\langle\partial^{\alpha-\beta} \sigma_{s t} \partial^{\beta} \nabla \cdot \bar{v},\left(1+\sigma_{s t}+\Phi\left(\sigma_{s t}\right)\right) \partial^{\alpha} \bar{\sigma}\right\rangle \\
& +\left\langle\partial^{\alpha-\beta} \bar{v} \cdot \partial^{\beta} \nabla \sigma_{s t},\left(1+\sigma_{s t}+\Phi\left(\sigma_{s t}\right)\right) \partial^{\alpha} \bar{\sigma}\right\rangle,
\end{aligned}
$$




$$
\begin{aligned}
I_{\alpha, \beta}^{(v)}(t)= & \left\langle\partial^{\alpha-\beta} \bar{v} \cdot \nabla \partial^{\beta} \bar{v},\left(1+\sigma_{s t}+\Phi\left(\sigma_{s t}\right)\right) \partial^{\alpha} \bar{v}\right\rangle \\
& +\frac{\gamma-1}{2}\left\langle\partial^{\alpha-\beta} \bar{\sigma} \nabla \partial^{\beta} \bar{\sigma},\left(1+\sigma_{s t}+\Phi\left(\sigma_{s t}\right)\right) \partial^{\alpha} \bar{v}\right\rangle \\
& +\frac{\gamma-1}{2}\left\langle\partial^{\alpha-\beta} \sigma_{s t} \nabla \partial^{\beta} \bar{\sigma},\left(1+\sigma_{s t}+\Phi\left(\sigma_{s t}\right)\right) \partial^{\alpha} \bar{v}\right\rangle \\
& +\left\langle\partial^{\alpha-\beta} \bar{v} \times \partial^{\beta} \bar{B},\left(1+\sigma_{s t}+\Phi\left(\sigma_{s t}\right)\right) \partial^{\alpha} \bar{v}\right\rangle \\
& +\frac{\gamma-1}{2}\left\langle\partial^{\alpha-\beta} \bar{\sigma} \nabla \partial^{\beta} \sigma_{s t},\left(1+\sigma_{s t}+\Phi\left(\sigma_{s t}\right)\right) \partial^{\alpha} \bar{v}\right\rangle
\end{aligned}
$$

and

$$
\begin{aligned}
I_{1}(t)= & \frac{1}{2}\left\langle\nabla \cdot \bar{v},\left(1+\sigma_{s t}+\Phi\left(\sigma_{s t}\right)\right)\left(\left|\partial^{\alpha} \bar{\sigma}\right|^{2}+\left|\partial^{\alpha} \bar{v}\right|^{2}\right)\right\rangle \\
& +\frac{\gamma-1}{2}\left\langle\nabla \bar{\sigma} \cdot \partial^{\alpha} \bar{v},\left(1+\sigma_{s t}+\Phi\left(\sigma_{s t}\right)\right) \partial^{\alpha} \bar{\sigma}\right\rangle-\left\langle\bar{v} \times \partial^{\alpha} \bar{B},\left(1+\sigma_{s t}+\Phi\left(\sigma_{s t}\right)\right) \partial^{\alpha} \bar{v}\right\rangle \\
& +\frac{\gamma-1}{2}\left\langle\nabla \sigma_{s t} \partial^{\alpha} \bar{v},\left(1+\sigma_{s t}+\Phi\left(\sigma_{s t}\right)\right) \partial^{\alpha} \bar{\sigma}\right\rangle-\frac{\gamma-1}{2}\left\langle\bar{\sigma} \partial^{\alpha} \nabla \sigma_{s t},\left(1+\sigma_{s t}+\Phi\left(\sigma_{s t}\right)\right) \partial^{\alpha} \bar{v}\right\rangle \\
& -\left\langle\bar{v} \cdot \partial^{\alpha} \nabla \sigma_{s t},\left(1+\sigma_{s t}+\Phi\left(\sigma_{s t}\right)\right) \partial^{\alpha} \bar{\sigma}\right\rangle \\
& +\left\langle\left(\frac{\gamma-1}{2} \bar{\sigma}+1\right) \partial^{\alpha} \bar{v}, \nabla\left(1+\sigma_{s t}+\Phi\left(\sigma_{s t}\right)\right) \partial^{\alpha} \bar{\sigma}\right\rangle \\
& +\frac{\gamma-1}{2}\left\langle\sigma_{s t} \partial^{\alpha} \bar{v}, \nabla\left(1+\sigma_{s t}+\Phi\left(\sigma_{s t}\right)\right) \partial^{\alpha} \bar{\sigma}\right\rangle \\
& +\frac{1}{2}\left\langle\bar{v}, \nabla\left(1+\sigma_{s t}+\Phi\left(\sigma_{s t}\right)\right)\left(\left|\partial^{\alpha} \bar{\sigma}\right|^{2}+\left|\partial^{\alpha} \bar{v}\right|^{2}\right)\right\rangle \triangleq \sum_{j=1}^{9} I_{1, j}(t) .
\end{aligned}
$$

When $|\alpha|=0$, it suffices to estimate $I_{1}(t)$ by

$$
\begin{aligned}
I_{1}(t) \leq & C\|\nabla \cdot \bar{v}\|\left(\|\bar{v}\|_{L^{6}}\|\bar{v}\|_{L^{3}}+\|\bar{\sigma}\|_{L^{6}}\|\bar{\sigma}\|_{L^{3}}\right)+C\|\nabla \bar{\sigma}\|\|\bar{v}\|_{L^{6}}\|\bar{\sigma}\|_{L^{3}}+C\|\bar{B}\|_{L^{\infty}}\|\bar{v}\|^{2} \\
& +C\left\|\nabla \sigma_{s t}\right\|\|\bar{\sigma}\|_{L^{6}}\|\bar{v}\|_{L^{3}}+C\left\|_{s t}\right\|_{L^{\infty}}\left\|\nabla \sigma_{s t}\right\|\|\bar{\sigma}\|_{L^{6}}\|\bar{v}\|_{L^{3}} \\
& +\|\bar{v}\|_{L^{\infty}}\left\|\nabla \sigma_{s t}\right\|\left(\|\bar{\sigma}\|_{L^{6}}\|\bar{\sigma}\|_{L^{3}}+\|\bar{v}\|_{L^{6}}\|\bar{v}\|_{L^{3}}\right) \\
\leq & C\left(\|[\bar{\sigma}, \bar{v}]\|_{H^{1}}+\delta+\delta\|\nabla \bar{v}\|_{H^{1}}\left(\|\nabla[\bar{\sigma}, \bar{v}]\|^{2}+\|[\bar{\sigma}, \bar{v}]\|^{2}\right)+C\|\nabla \bar{B}\|_{H^{1}}\|\bar{v}\|^{2},\right.
\end{aligned}
$$

which is further bounded by the r.h.s. term of (3.23). When $|\alpha| \geq 1$, for $I_{1}(t)$, the similarity of $I_{1,1}(t)$ and $I_{1,2}(t)$ shows that we can estimate them together as follows

$$
\begin{aligned}
I_{1,1}(t)+I_{1,2}(t) \leq & C\|\nabla \cdot \bar{v}\|_{L^{\infty}}\left\|\left(1+\sigma_{s t}+\Phi\left(\sigma_{s t}\right)\right)\right\|_{L^{\infty}}\|\nabla[\bar{\sigma}, \bar{v}]\|_{N-1}^{2} \\
& +C\|\nabla \bar{\sigma}\|_{L^{\infty}}\left\|\left(1+\sigma_{s t}+\Phi\left(\sigma_{s t}\right)\right)\right\|_{L^{\infty}}\|\nabla[\bar{\sigma}, \bar{v}]\|_{N-1}^{2} \\
\leq & C\|[\bar{\sigma}, \bar{v}]\|_{N}\|\nabla[\bar{\sigma}, \bar{v}]\|_{N-1}^{2} .
\end{aligned}
$$

For $I_{1,3}(t), I_{1,5}(t)$ and $I_{1,6}(t)$, there are no derivative of $\bar{\sigma}$ or $\bar{v}$, then we use $L^{\infty}$ of $\bar{v}$ or $\bar{\sigma}$,

$$
\begin{aligned}
I_{1,3}(t)+I_{1,5}(t)+I_{1,6}(t) \leq & C\|\bar{v}\|_{L^{\infty}}\left\|\partial^{\alpha} \bar{B}\right\|\left\|\left(1+\sigma_{s t}+\Phi\left(\sigma_{s t}\right)\right)\right\|_{L^{\infty}}\|\nabla \bar{v}\|_{N-1} \\
& +C\|\bar{\sigma}\|_{L^{\infty}}\left\|\partial^{\alpha} \nabla \sigma_{s t}\right\|\left\|\left(1+\sigma_{s t}+\Phi\left(\sigma_{s t}\right)\right)\right\|_{L^{\infty}}\|\nabla \bar{v}\|_{N-1} \\
& +C\|\bar{v}\|_{L^{\infty}}\left\|\partial^{\alpha} \nabla \sigma_{s t}\right\|\left\|\left(1+\sigma_{s t}+\Phi\left(\sigma_{s t}\right)\right)\right\|_{L^{\infty}}\|\nabla \bar{\sigma}\|_{N-1} \\
\leq & C\left(\delta+\|\bar{B}\|_{N}\right)\|\nabla[\bar{\sigma}, \bar{v}]\|_{N-1}^{2} .
\end{aligned}
$$

For other terms of $I_{1}(t)$, both $\bar{\sigma}$ and $\bar{v}$ contain the derivative, one can use the $L^{2}$ of these terms and $L^{\infty}$ of others. Combining the above two estimates, one has

$$
I_{1}(t) \leq C\left(\|[\bar{\sigma}, \bar{v}, \bar{B}]\|_{N}+\delta+\delta\|\nabla \bar{v}\|_{H^{1}}\right)\|\nabla[\bar{\sigma}, \bar{v}]\|_{N-1}^{2},
$$


which is bounded by the r.h.s. term of (3.23). On the other hand, since each term in $I_{\alpha, \beta}(t)$ is the integration of the four-terms product in which there is at least one term containing the derivative, one has

$$
I_{\alpha, \beta}(t) \leq C\left(\|[\bar{\sigma}, \bar{v}, \bar{B}]\|_{N}+\delta+\delta\|\nabla \bar{v}\|_{H^{1}}\right)\|\nabla[\bar{\sigma}, \bar{v}]\|_{N-1}^{2},
$$

which is also bounded by the r.h.s. term of (3.23).

From (3.8), energy estimates on $\partial^{\alpha} \bar{E}$ and $\partial^{\alpha} \bar{B}$ with $|\alpha| \leq N$ give

$$
\begin{aligned}
& \frac{1}{2} \frac{d}{d t}\left\|\partial^{\alpha}[\bar{E}, \bar{B}]\right\|^{2}-\frac{1}{\sqrt{\gamma}}\left\langle\left(1+\sigma_{s t}+\Phi\left(\sigma_{s t}\right)\right) \partial^{\alpha} \bar{v}, \partial^{\alpha} \bar{E}\right\rangle \\
= & \frac{1}{\sqrt{\gamma}}\left\langle\partial^{\alpha}\left[\left(\Phi\left(\bar{\sigma}+\sigma_{s t}\right)-\Phi\left(\sigma_{s t}\right)\right) \bar{v}\right], \partial^{\alpha} \bar{E}\right\rangle+\frac{1}{\sqrt{\gamma}}\left\langle\partial^{\alpha}[\bar{\sigma} \bar{v}], \partial^{\alpha} \bar{E}\right\rangle \\
& +\frac{1}{\sqrt{\gamma}} \sum_{\beta<\alpha} C_{\beta}^{\alpha}\left\langle\partial^{\alpha-\beta}\left(1+\sigma_{s t}+\Phi\left(\sigma_{s t}\right)\right) \partial^{\beta} \bar{v}, \partial^{\alpha} \bar{E}\right\rangle \\
= & I_{2,1}(t)+I_{2,2}(t)+\sum_{\beta<\alpha} C_{\beta}^{\alpha} I_{2, \beta}(t) .
\end{aligned}
$$

In a similar way as before, when $|\alpha|=0$, it suffices to estimate $I_{2,1}(t)+I_{2,2}(t)$ by

$$
I_{2,1}(t)+I_{2,2}(t) \leq C\|\nabla \bar{\sigma}\| \cdot\|\bar{v}\|_{1}\|\bar{E}\| .
$$

When $|\alpha|>0, I_{2,1}(t)$ and $I_{2,2}(t)$ can be estimated in a similar way as in [5],

$$
I_{2,1}(t)+I_{2,2}(t) \leq C\|\nabla \bar{\sigma}\|_{N-1}\|\nabla \bar{v}\|_{N-1}\|\bar{E}\|_{N} .
$$

When $|\alpha|>0$, for each $\beta$ with $\beta<\alpha, I_{2, \beta}$ is estimated by three cases.

Case 1. $|\alpha|=N$. In this case, integration by parts shows that

$$
\begin{aligned}
I_{2, \beta}(t) & \leq C \delta\|\nabla \bar{v}\|_{N-1}\|\nabla \bar{E}\|_{N-2} \\
& \leq C \delta\|\nabla \bar{v}\|_{N-1}^{2}+C \delta\|\nabla \bar{E}\|_{N-2}^{2} .
\end{aligned}
$$

Case 2. $|\alpha|<N$ and $|\beta| \geq 1$ which imply $|\alpha-\beta| \leq N-2$. It holds that

$$
\begin{aligned}
I_{2, \beta}(t) & \leq C\left\|\partial^{\alpha-\beta}\left(1+\sigma_{s t}+\Phi\left(\sigma_{s t}\right)\right)\right\|_{L^{\infty}}\left\|\partial^{\beta} \bar{v}\right\|\left\|\partial^{\alpha} \bar{E}\right\| \\
& \leq C\left\|\nabla \partial^{\alpha-\beta}\left(1+\sigma_{s t}+\Phi\left(\sigma_{s t}\right)\right)\right\|_{H^{1}}\|\nabla \bar{v}\|_{N-1}\|\nabla \bar{E}\|_{N-2} \\
& \leq C \delta\|\nabla \bar{v}\|_{N-1}^{2}+C \delta\|\nabla \bar{E}\|_{N-2}^{2} .
\end{aligned}
$$

Case 3. $|\alpha|<N$ and $|\beta|=0$. In this case, there is no derivative of $\bar{v}$, one can use $L^{\infty}$ of $\bar{v}$ to estimate $I_{2, \beta}(t)$,

$$
\begin{aligned}
I_{2, \beta}(t) & \leq C\left\|\partial^{\alpha-\beta}\left(1+\sigma_{s t}+\Phi\left(\sigma_{s t}\right)\right)\right\|\|\bar{v}\|_{L^{\infty}}\left\|\partial^{\alpha} \bar{E}\right\| \\
& \leq C \delta\|\nabla \bar{v}\|_{N-1}^{2}+C \delta\|\nabla \bar{E}\|_{N-2}^{2},
\end{aligned}
$$

which is bounded by the r.h.s. term of (3.23). Then (3.23) follows by taking summation of (3.24) and (3.25) over $|\alpha| \leq N$. Then the time evolution of the full instant energy $\|V(t)\|_{N}^{2}$ has been obtained but its dissipation rate only contains the contribution from the explicit relaxation variable $\bar{v}$. In a parallel way as 5 , by introducing some interactive functionals, the dissipation from contributions of the rest components $\bar{\sigma}, \bar{E}$, and $\bar{B}$ can be recovered in turn.

Step 2. It holds that

$$
\begin{aligned}
& \frac{d}{d t} \mathcal{E}_{N, 1}^{i n t}(\bar{V})+\lambda\|\bar{\sigma}\|_{N}^{2} \\
\leq & C\|\nabla \bar{v}\|_{N-1}^{2}+C\left(\|[\bar{\sigma}, \bar{v}, \bar{B}]\|_{N}^{2}+\delta\right)\|\nabla[\bar{\sigma}, \bar{v}]\|_{N-1}^{2},
\end{aligned}
$$


where $\mathcal{E}_{N, 1}^{\text {int }}(\cdot)$ is defined by

$$
\mathcal{E}_{N, 1}^{i n t}(\bar{V})=\sum_{|\alpha| \leq N-1}\left\langle\partial^{\alpha} \bar{v}, \nabla \partial^{\alpha} \bar{\sigma}\right\rangle
$$

In fact, the first two equations of (3.8) can be rewritten as

$$
\begin{gathered}
\partial_{t} \bar{\sigma}+\nabla \cdot \bar{v}=f_{1}, \\
\partial_{t} \bar{v}+\nabla \bar{\sigma}+\frac{1}{\sqrt{\gamma}} \bar{E}=f_{2}-\frac{1}{\sqrt{\gamma}} \bar{v}
\end{gathered}
$$

where

$$
\left\{\begin{array}{l}
f_{1}:=-\bar{v} \cdot \nabla \bar{\sigma}-\frac{\gamma-1}{2} \bar{\sigma} \nabla \cdot \bar{v}-\bar{v} \cdot \nabla \sigma_{s t}-\frac{\gamma-1}{2} \sigma_{s t} \nabla \cdot \bar{v}, \\
f_{2}:=-\bar{v} \cdot \nabla \bar{v}-\frac{\gamma-1}{2} \bar{\sigma} \nabla \bar{\sigma}-\bar{v} \times \bar{B}-\frac{\gamma-1}{2} \sigma_{s t} \nabla \bar{\sigma}-\frac{\gamma-1}{2} \bar{\sigma} \nabla \sigma_{s t} .
\end{array}\right.
$$

Let $|\alpha| \leq N-1$. Applying $\partial^{\alpha}$ to (3.28), multiplying it by $\partial^{\alpha} \nabla \bar{\sigma}$, taking integrations in $x$ and then using integration by parts and also the final equation of (3.8), replacing $\partial_{t} \bar{\sigma}$ from (3.27) give

$$
\begin{aligned}
& \frac{d}{d t}\left\langle\partial^{\alpha} \bar{v}, \nabla \partial^{\alpha} \bar{\sigma}\right\rangle+\left\|\nabla \partial^{\alpha} \bar{\sigma}\right\|^{2}+\frac{1}{\gamma}\left\|\partial^{\alpha} \bar{\sigma}\right\|^{2} \\
= & -\frac{1}{\gamma}\left\langle\partial^{\alpha}\left(\Phi\left(\bar{\sigma}+\sigma_{s t}\right)-\Phi\left(\sigma_{s t}\right)\right), \partial^{\alpha} \bar{\sigma}\right\rangle+\left\langle\partial^{\alpha} f_{2}, \nabla \partial^{\alpha} \bar{\sigma}\right\rangle \\
& -\frac{1}{\sqrt{\gamma}}\left\langle\partial^{\alpha} \bar{v}, \nabla \partial^{\alpha} \bar{\sigma}\right\rangle+\left\|\nabla \cdot \partial^{\alpha} \bar{v}\right\|^{2}-\left\langle\partial^{\alpha} f_{1}, \nabla \cdot \partial^{\alpha} \bar{v}\right\rangle .
\end{aligned}
$$

Then, it follows from Cauchy-Schwarz inequality that

$$
\begin{aligned}
& \frac{d}{d t}\left\langle\partial^{\alpha} \bar{v}, \nabla \partial^{\alpha} \bar{\sigma}\right\rangle+\lambda\left(\left\|\nabla \partial^{\alpha} \bar{\sigma}\right\|^{2}+\left\|\partial^{\alpha} \bar{\sigma}\right\|^{2}\right) \\
\leq & C\left\|\nabla \cdot \partial^{\alpha} \bar{v}\right\|^{2}+C\left(\left\|\partial^{\alpha}\left(\Phi\left(\bar{\sigma}+\sigma_{s t}\right)-\Phi\left(\sigma_{s t}\right)\right)\right\|^{2}+\left\|\partial^{\alpha} f_{1}\right\|^{2}+\left\|\partial^{\alpha} f_{2}\right\|^{2}\right) .
\end{aligned}
$$

Noticing that $\Phi(\sigma)$ is smooth in $\sigma$ with $\Phi^{\prime}(0)=0$, one has from (3.29) that

$$
\begin{aligned}
& \left\|\partial^{\alpha}\left(\Phi\left(\bar{\sigma}+\sigma_{s t}\right)-\Phi\left(\sigma_{s t}\right)\right)\right\|^{2}+\left\|\partial^{\alpha} f_{1}\right\|^{2}+\left\|\partial^{\alpha} f_{2}\right\|^{2} \\
\leq & C\left(\|[\bar{\sigma}, \bar{v}, \bar{B}]\|_{N}^{2}+\delta\right)\|\nabla[\bar{\sigma}, \bar{v}]\|_{N-1}^{2} .
\end{aligned}
$$

Here, if there is no derivative on $\bar{\sigma}$ or $\bar{v}$, then use the $L^{\infty}$ of $\bar{\sigma}$ or $\bar{v}$. Plugging this into (3.30) taking summation over $|\alpha| \leq N-1$ yield (3.26).

Step 3. It holds that

$$
\begin{aligned}
\frac{d}{d t} \mathcal{E}_{N, 2}^{i n t}(\bar{V})+\lambda\|\bar{E}\|_{N-1}^{2} \leq C & \|[\bar{\sigma}, \bar{v}]\|_{N}^{2}+C\|\bar{v}\|_{N}\|\nabla \bar{B}\|_{N-2} \\
& +C\left(\|[\bar{\sigma}, \bar{v}, \bar{B}]\|_{N}^{2}+\delta\right)\|\nabla[\bar{\sigma}, \bar{v}]\|_{N-1}^{2},
\end{aligned}
$$

where $\mathcal{E}_{N, 2}^{i n t}(\cdot)$ is defined by

$$
\mathcal{E}_{N, 2}^{i n t}(\bar{V})=\sum_{|\alpha| \leq N-1}\left\langle\partial^{\alpha} \bar{v}, \partial^{\alpha} \bar{E}\right\rangle
$$


Applying $\partial^{\alpha}$ to (3.28), multiplying it by $\partial^{\alpha} \bar{E}$, taking integrations in $x$ and using integration by parts and replacing $\partial_{t} \bar{E}$ from the third equation of (3.8) give

$$
\begin{aligned}
& \frac{d}{d t}\left\langle\partial^{\alpha} \bar{v}, \partial^{\alpha} \bar{E}\right\rangle+\frac{1}{\sqrt{\gamma}}\left\|\partial^{\alpha} \bar{E}\right\|^{2} \\
= & \frac{1}{\sqrt{\gamma}}\left\|\partial^{\alpha} \bar{v}\right\|^{2}+\frac{1}{\sqrt{\gamma}}\left\langle\partial^{\alpha} \bar{v}, \nabla \times \partial^{\alpha} \bar{B}\right\rangle+\frac{1}{\sqrt{\gamma}}\left\langle\partial^{\alpha} \bar{v}, \partial^{\alpha}\left[\Phi\left(\bar{\sigma}+\sigma_{s t}\right) \bar{v}+\left(\bar{\sigma}+\sigma_{s t}\right) \bar{v}\right]\right\rangle \\
& -\left\langle\partial^{\alpha} \nabla \bar{\sigma}+\frac{1}{\sqrt{\gamma}} \partial^{\alpha} \bar{v}, \partial^{\alpha} \bar{E}\right\rangle+\left\langle\partial^{\alpha} f_{2}, \partial^{\alpha} \bar{E}\right\rangle,
\end{aligned}
$$

which from the Cauchy-Schwarz inequality further implies

$$
\begin{aligned}
& \frac{d}{d t}\left\langle\partial^{\alpha} \bar{v}, \partial^{\alpha} \bar{E}\right\rangle+\lambda\left\|\partial^{\alpha} \bar{E}\right\|^{2} \\
\leq & C\|[\bar{\sigma}, \bar{v}]\|_{N}^{2}+C\|\bar{v}\|_{N}\|\nabla \bar{B}\|_{N-2}+C\left(\|[\bar{\sigma}, \bar{v}, \bar{B}]\|_{N}^{2}+\delta\right)\|\nabla[\bar{\sigma}, \bar{v}]\|_{N-1}^{2} .
\end{aligned}
$$

Thus (3.31) follows from taking summation of the above estimate over $|\alpha| \leq N-1$.

Step 4. It holds that

$$
\begin{aligned}
\frac{d}{d t} \mathcal{E}_{N, 3}^{i n t}(\bar{V})+\lambda\|\nabla \bar{B}\|_{N-2}^{2} \leq & C\|[\bar{v}, \bar{E}]\|_{N-1}^{2} \\
& +C\left(\|\bar{\sigma}\|_{N}^{2}+\delta\right)\|\nabla \bar{v}\|_{N-1}^{2},
\end{aligned}
$$

where $\mathcal{E}_{N, 3}^{i n t}(\cdot)$ is defined by

$$
\mathcal{E}_{N, 3}^{i n t}(\bar{V})=-\sum_{|\alpha| \leq N-2}\left\langle\nabla \times \partial^{\alpha} \bar{E}, \partial^{\alpha} \bar{B}\right\rangle .
$$

In fact, for $|\alpha| \leq N-2$, applying $\partial^{\alpha}$ to the third equation of (3.8), multiplying it by $-\partial^{\alpha} \nabla \times \bar{B}$, taking integrations in $x$ and using integration by parts and replacing $\partial_{t} \bar{B}$ from the fourth equation of (3.8) implie

$$
\begin{aligned}
& -\frac{d}{d t}\left\langle\partial^{\alpha} \bar{E}, \nabla \times \partial^{\alpha} \bar{B}\right\rangle+\frac{1}{\sqrt{\gamma}}\left\|\nabla \times \partial^{\alpha} \bar{B}\right\|^{2} \\
= & \frac{1}{\sqrt{\gamma}}\left\|\nabla \times \partial^{\alpha} \bar{E}\right\|^{2}-\frac{1}{\sqrt{\gamma}}\left\langle\partial^{\alpha} \bar{v}, \nabla \times \partial^{\alpha} \bar{B}\right\rangle-\frac{1}{\sqrt{\gamma}}\left\langle\partial^{\alpha}\left[\Phi\left(\bar{\sigma}+\sigma_{s t}\right) \bar{v}+\left(\bar{\sigma}+\sigma_{s t}\right) \bar{v}\right], \nabla \times \partial^{\alpha} \bar{B}\right\rangle,
\end{aligned}
$$

which gives (3.32) by further using Cauchy-Schwarz inequality and taking summation over $|\alpha| \leq N-2$, where we also used

$$
\left\|\partial^{\alpha} \partial_{i} \bar{B}\right\|=\left\|\partial_{i} \Delta^{-1} \nabla \times\left(\nabla \times \partial^{\alpha} \bar{B}\right)\right\| \leq\left\|\nabla \times \partial^{\alpha} \bar{B}\right\|
$$

for each $1 \leq i \leq 3$, due to the fact $\partial_{i} \Delta^{-1} \nabla$ is bounded from $L^{p}$ to itself for $1<p<\infty$, cf. 23].

Step 5. Now, following the four steps above, we are ready to prove (3.22). Let us define

$\mathcal{E}_{N}(\bar{V}(t))=\sum_{|\alpha| \leq N} \int_{\mathbb{R}^{3}}\left(1+\sigma_{s t}+\Phi\left(\sigma_{s t}\right)\right)\left(\left|\partial^{\alpha} \bar{\sigma}\right|^{2}+\left|\partial^{\alpha} \bar{v}\right|^{2}\right) d x+\|[\bar{E}, \bar{B}]\|_{N}^{2}+\sum_{i=1}^{3} \kappa_{i} \mathcal{E}_{N, i}^{i n t}(\bar{V}(t))$,

that is,

$$
\begin{aligned}
\mathcal{E}_{N}(\bar{V}(t))= & \sum_{|\alpha| \leq N} \int_{\mathbb{R}^{3}}\left(1+\sigma_{s t}+\Phi\left(\sigma_{s t}\right)\right)\left(\left|\partial^{\alpha} \bar{\sigma}\right|^{2}+\left|\partial^{\alpha} \bar{v}\right|^{2}\right) d x+\|[\bar{E}, \bar{B}]\|_{N}^{2} \\
& +\kappa_{1} \sum_{|\alpha| \leq N-1}\left\langle\partial^{\alpha} \bar{v}, \nabla \partial^{\alpha} \bar{\sigma}\right\rangle+\kappa_{2} \sum_{|\alpha| \leq N-1}\left\langle\partial^{\alpha} \bar{v}, \partial^{\alpha} \bar{E}\right\rangle \\
& -\kappa_{3} \sum_{|\alpha| \leq N-2}\left\langle\nabla \times \partial^{\alpha} \bar{E}, \partial^{\alpha} \bar{B}\right\rangle
\end{aligned}
$$


for constants $0<\kappa_{3} \ll \kappa_{2} \ll \kappa_{1} \ll 1$ to be determined. Notice that as long as $0<\kappa_{i} \ll 1$ is small enough for $i=1,2,3$, and $\sigma_{s t}+\Phi\left(\sigma_{s t}\right)$ depending only on $x$ is sufficiently small compared with 1 , then $\mathcal{E}_{N}(\bar{V}(t)) \sim\|\bar{V}(t)\|_{N}^{2}$ holds true. Moreover, letting $0<\kappa_{3} \ll \kappa_{2} \ll \kappa_{1} \ll 1$ with $\kappa_{2}^{3 / 2} \ll \kappa_{3}$, the sum of $(3.23) \times \kappa_{1},(3.26) \times \kappa_{2},(3.32) \times \kappa_{3}$ implies that there are $\lambda>0, C>0$ such that (3.22) holds true with $\mathcal{D}_{N}(\cdot)$ defined in (3.13). Here, we have used the following Cauchy-Schwarz inequality:

$$
2 \kappa_{2}\|\bar{v}\|_{N}\|\nabla \bar{B}\|_{N-2} \leq \kappa_{2}^{1 / 2}\|\bar{v}\|_{N}^{2}+\kappa_{2}^{3 / 2}\|\nabla \bar{B}\|_{N-2}^{2}
$$

Due to $\kappa_{2}^{3 / 2} \ll \kappa_{3}$, both terms on the r.h.s. of the above inequality were absorbed. This completes the proof of Theorem 3.1 .

Since (3.8) is a quasi-linear symmetric hyperbolic system, the short-time existence can be proved in much more general case as in [16]; see also (Theorem 1.2, Proposition 1.3, and Proposition 1.4 in Chapter 16 of [24]). From Theorem 3.1 and the continuity argument, it is easy to see that $\mathcal{E}_{N}(\bar{V}(t))$ is bounded uniformly in time under the assumptions that $\mathcal{E}_{N}\left(\bar{V}_{0}\right)>0$ and $\left\|n_{b}-1\right\|_{W_{0}^{N+1,2}}$ are small enough. Therefore, the global existence of solutions satisfying (3.15) and (3.16) follows in the standard way; see also [5]. This completes the proof of Proposition 3.1 .

\section{DeCAy in time FOR THE NON-LINEAR SyStem}

In this section, we are devoted to the rate of the convergence of solution to the equilibrium $\left[n_{s t}, 0, E_{s t}, 0\right]$ for the system (1.1) over $\mathbb{R}^{3}$. In fact by setting

$$
\bar{\rho}=n-n_{s t}, \quad \bar{u}=u, \quad E_{1}=E-E_{s t}, \quad B_{1}=B,
$$

and

$$
\rho_{s t}=n_{s t}-1
$$

then $\bar{U}:=\left[\bar{\rho}, \bar{u}, E_{1}, B_{1}\right]$ satisfies

$$
\left\{\begin{array}{l}
\partial_{t} \bar{\rho}+\nabla \cdot \bar{u}=g_{1}, \\
\partial_{t} \bar{u}+\bar{u}+E_{1}+\gamma \nabla \bar{\rho}=g_{2}, \\
\partial_{t} E_{1}-\nabla \times B_{1}-\bar{u}=g_{3}, \\
\partial_{t} B_{1}+\nabla \times E_{1}=0, \\
\nabla \cdot E_{1}=-\bar{\rho}, \quad \nabla \cdot B_{1}=0, \quad t>0, x \in \mathbb{R}^{3},
\end{array}\right.
$$

with initial data

$$
\begin{aligned}
\left.\bar{U}\right|_{t=0}=\bar{U}_{0}: & =\left[\bar{\rho}_{0}, \bar{u}_{0}, E_{1,0}, B_{1,0}\right] \\
& =\left[n_{0}-n_{s t}, u_{0}, E_{0}-E_{s t}, B_{0}\right], \quad x \in \mathbb{R}^{3},
\end{aligned}
$$

satisfying the compatible conditions

$$
\nabla \cdot E_{1,0}=-\bar{\rho}_{0}, \quad \nabla \cdot B_{1,0}=0 .
$$

Here the nonlinear source terms take the form of

$$
\left\{\begin{aligned}
g_{1}= & -\nabla \cdot\left[\left(\bar{\rho}+\rho_{s t}\right) \bar{u}\right], \\
g_{2}= & -\bar{u} \cdot \nabla \bar{u}-\bar{u} \times B_{1}-\gamma\left[\left(\bar{\rho}+1+\rho_{s t}\right)^{\gamma-2}-1\right] \nabla \bar{\rho} \\
& -\gamma\left[\left(1+\bar{\rho}+\rho_{s t}\right)^{\gamma-2}-\left(1+\rho_{s t}\right)^{\gamma-2}\right] \nabla \rho_{s t}, \\
g_{3}= & \left(\bar{\rho}+\rho_{s t}\right) \bar{u} .
\end{aligned}\right.
$$


In what follows, we will denote $[\rho, u, E, B]$ as the solution to the the following linearized equation of (4.1):

$$
\left\{\begin{array}{l}
\partial_{t} \rho+\nabla \cdot u=0 \\
\partial_{t} u+u+E+\gamma \nabla \rho=0, \\
\partial_{t} E-\nabla \times B-u=0, \\
\partial_{t} B+\nabla \times E=0, \\
\nabla \cdot E=-\rho, \quad \nabla \cdot B=0, \quad t>0, \quad x \in \mathbb{R}^{3},
\end{array}\right.
$$

with given initial data

$$
\left.U\right|_{t=0}=\bar{U}_{0}:=\left[\bar{\rho}_{0}, \bar{u}_{0}, E_{1,0}, B_{1,0}\right], \quad x \in \mathbb{R}^{3},
$$

satisfying the compatible conditions (4.3).

For the above linearized equations, the $L^{p}-L^{q}$ time-decay property was proved by Duan in [5]. We list only some special $L^{p}-L^{q}$ time decay properties in the following proposition.

Proposition 4.1. Suppose $U(t)=e^{t L} \bar{U}_{0}$ is the solution to the Cauchy problem (4.5)-(4.6) with the initial data $\bar{U}_{0}=\left[\bar{\rho}_{0}, \bar{u}_{0}, E_{1,0}, B_{1,0}\right]$ satisfying (4.3). Then, $U=[\rho, u, E, B]$ satisfies the following time-decay property:

$$
\left\{\begin{array}{l}
\|\rho(t)\| \leq C e^{-\frac{t}{2}}\left\|\left[\bar{\rho}_{0}, \bar{u}_{0}\right]\right\|, \\
\|u(t)\| \leq C e^{-\frac{t}{2}}\left\|\bar{\rho}_{0}\right\|+C(1+t)^{-\frac{5}{4}}\left\|\left[\bar{u}_{0}, E_{1,0}, B_{1,0}\right]\right\|_{L^{1} \cap \dot{H}^{2}}, \\
\|E(t)\| \leq C(1+t)^{-\frac{5}{4}}\left\|\left[\bar{u}_{0}, E_{1,0}, B_{1,0}\right]\right\|_{L^{1} \cap \dot{H}^{3}}, \\
\|B(t)\| \leq C(1+t)^{-\frac{3}{4}}\left\|\left[\bar{u}_{0}, E_{1,0}, B_{1,0}\right]\right\|_{L^{1} \cap \dot{H}^{2}},
\end{array}\right.
$$

and

$$
\left\{\begin{array}{l}
\|\rho(t)\|_{\infty} \leq C e^{-\frac{t}{2}}\left\|\left[\bar{\rho}_{0}, \bar{u}_{0}\right]\right\|_{L^{2} \cap \dot{H}^{2}}, \\
\|u(t)\|_{\infty} \leq C e^{-\frac{t}{2}}\left\|\bar{\rho}_{0}\right\|_{L^{2} \cap \dot{H}^{2}}+C(1+t)^{-2}\left\|\left[\bar{u}_{0}, E_{1,0}, B_{1,0}\right]\right\|_{L^{1} \cap \dot{H}^{5}}, \\
\|E(t)\|_{\infty} \leq C(1+t)^{-2}\left\|\left[\bar{u}_{0}, E_{1,0}, B_{1,0}\right]\right\|_{L^{1} \cap \dot{H}^{6}}, \\
\|B(t)\|_{\infty} \leq C(1+t)^{-\frac{3}{2}}\left\|\left[\bar{u}_{0}, E_{1,0}, B_{1,0}\right]\right\|_{L^{1} \cap \dot{H}^{5}},
\end{array}\right.
$$

and, moreover,

$$
\left\{\begin{array}{l}
\|\nabla B(t)\| \leq C(1+t)^{-\frac{5}{4}}\left\|\left[\bar{u}_{0}, E_{1,0}, B_{1,0}\right]\right\|_{L^{1} \cap \dot{H}^{4}}, \\
\left\|\nabla^{N}[E(t), B(t)]\right\| \leq C(1+t)^{-\frac{5}{4}}\left\|\left[\bar{u}_{0}, E_{1,0}, B_{1,0}\right]\right\|_{L^{1} \cap \dot{H}^{N+3}} .
\end{array}\right.
$$

In what follows, since we shall apply the linear $L^{p}-L^{q}$ time-decay property of the homogeneous system (4.5), we need the mild form of the non-linear Cauchy problem (4.1)-(4.2). From now on, we always denote $\bar{U}=\left[\bar{\rho}, \bar{u}, E_{1}, B_{1}\right]$ to the non-linear Cauchy problem (4.1)-(4.2). Then, by Duhamel's principle, the solution $\bar{U}$ can be formally written as

$$
\bar{U}(t)=e^{t L} \bar{U}_{0}+\int_{0}^{t} e^{(t-s) L}\left[g_{1}(s), g_{2}(s), g_{3}(s), 0\right] d s,
$$

where $e^{t L} \bar{U}_{0}$ denotes the solution to the Cauchy problem (4.5)-(4.6) without nonlinear sources.

The following two lemmas give the full and high-order energy estimates.

Lemma 4.1. Let $\bar{V}=[\bar{\sigma}, \bar{v}, \bar{E}, \bar{B}]$ be the solution to the Cauchy problem (3.8)-(3.9) with initial data $\bar{V}_{0}=\left[\bar{\sigma}_{0}, \bar{v}_{0}, \bar{E}_{0}, \bar{B}_{0}\right]$ satisfying (3.10). Then, if $\mathcal{E}_{N}\left(\bar{V}_{0}\right)$ and $\left\|n_{b}-1\right\|_{W_{0}^{N+1,2}}$ are sufficiently small,

$$
\frac{d}{d t} \mathcal{E}_{N}(\bar{V}(t))+\lambda \mathcal{D}_{N}(\bar{V}(t)) \leq 0
$$


holds for any $t>0$, where $\mathcal{E}_{N}(\bar{V}(t)), \mathcal{D}_{N}(\bar{V}(t))$ are defined in the form of (3.11) and (3.13), respectively.

Proof. It can be seen directly from the proof of Theorem 3.1

Lemma 4.2. Let $\bar{V}=[\bar{\sigma}, \bar{v}, \bar{E}, \bar{B}]$ be the solution to the Cauchy problem (3.8)-(3.9) with initial data $\bar{V}_{0}=\left[\bar{\sigma}_{0}, \bar{v}_{0}, \bar{E}_{0}, \bar{B}_{0}\right]$ satisfying (3.10) in the sense of Proposition 3.1. Then if $\mathcal{E}_{N}\left(\bar{V}_{0}\right)$ and $\left\|n_{b}-1\right\|_{W_{0}^{N+1,2}}$ are sufficiently small, there are the high-order instant energy functional $\mathcal{E}_{N}^{h}(\cdot)$ and the corresponding dissipation rate $\mathcal{D}_{N}^{h}(\cdot)$ such that

$$
\frac{d}{d t} \mathcal{E}_{N}^{h}(\bar{V}(t))+\lambda \mathcal{D}_{N}^{h}(\bar{V}(t)) \leq 0,
$$

holds for any $t \geq 0$.

Proof. The proof can be done by modifying the proof of Theorem 3.1 a little. In fact, by letting the energy estimates made only on the high-order derivatives, then corresponding to (3.23), (3.26), (3.31) and (3.32), it can be re-verified that

$$
\begin{gathered}
\frac{1}{2} \frac{d}{d t}\left(\sum_{1 \leq|\alpha| \leq N} \int_{\mathbb{R}^{3}}\left(1+\sigma_{s t}+\Phi\left(\sigma_{s t}\right)\right)\left(\left|\partial^{\alpha} \bar{\sigma}\right|^{2}+\left|\partial^{\alpha} \bar{v}\right|^{2}\right) d x+\|\nabla[\bar{E}, \bar{B}]\|_{N-1}^{2}\right) \\
\quad+\frac{1}{\sqrt{\gamma}} \sum_{1 \leq|\alpha| \leq N} \int_{\mathbb{R}^{3}}\left(1+\sigma_{s t}+\Phi\left(\sigma_{s t}\right)\right)\left|\partial^{\alpha} \bar{v}\right|^{2} d x \\
\leq C\left(\|\bar{V}\|_{N}+\delta\right)\left(\|\nabla[\bar{\sigma}, \bar{v}]\|_{N-1}^{2}+\|\nabla \bar{E}\|_{N-2}^{2}\right), \\
\frac{d}{d t} \sum_{1 \leq|\alpha| \leq N-1}\left\langle\partial^{\alpha} \bar{v}, \nabla \partial^{\alpha} \bar{\sigma}\right\rangle+\lambda\|\nabla \bar{\sigma}\|_{N-1}^{2} \leq C\left\|\nabla^{2} \bar{v}\right\|_{N-2}^{2}+C\left(\|[\bar{\sigma}, \bar{v}, \bar{B}]\|_{N}^{2}+\delta\right)\|\nabla[\bar{\sigma}, \bar{v}]\|_{N-1}^{2}, \\
\frac{d}{d t} \sum_{1 \leq|\alpha| \leq N-1}\left\langle\partial^{\alpha} \bar{v}, \partial^{\alpha} \bar{E}\right\rangle+\lambda\|\nabla \bar{E}\|_{N-2}^{2} \leq C\|\nabla[\bar{\sigma}, \bar{v}]\|_{N-1}^{2}+C\|\nabla \bar{v}\|_{N-1}\left\|\nabla^{2} \bar{B}\right\|_{N-3} \\
+C\left(\|[\bar{\sigma}, \bar{v}, \bar{B}]\|_{N}^{2}+\delta\right)\|\nabla[\bar{\sigma}, \bar{v}]\|_{N-1}^{2},
\end{gathered}
$$

and

$$
\begin{gathered}
-\frac{d}{d t} \sum_{1 \leq|\alpha| \leq N-2}\left\langle\nabla \times \partial^{\alpha} \bar{E}, \partial^{\alpha} \bar{B}\right\rangle+\lambda\left\|\nabla^{2} \bar{B}\right\|_{N-3}^{2} \\
\leq C\left\|\nabla^{2} \bar{E}\right\|_{N-3}^{2}+C\|\nabla \bar{v}\|_{N-3}^{2}+C\left(\|\bar{\sigma}\|_{N}^{2}+\delta\right)\|\nabla \bar{v}\|_{N-1}^{2} .
\end{gathered}
$$

Here, the details of proof are omitted for simplicity. Now, similar to (3.33), let us define

$$
\begin{aligned}
\mathcal{E}_{N}^{h}(\bar{V}(t)) & =\sum_{1 \leq|\alpha| \leq N} \int_{\mathbb{R}^{3}}\left(1+\sigma_{s t}+\Phi\left(\sigma_{s t}\right)\right)\left(\left|\partial^{\alpha} \bar{\sigma}\right|^{2}+\left|\partial^{\alpha} \bar{v}\right|^{2}\right) d x+\|\nabla[\bar{E}, \bar{B}]\|_{N-1}^{2} \\
& +\kappa_{1} \sum_{1 \leq|\alpha| \leq N-1}\left\langle\partial^{\alpha} \bar{v}, \nabla \partial^{\alpha} \bar{\sigma}\right\rangle+\kappa_{2} \sum_{1 \leq|\alpha| \leq N-1}\left\langle\partial^{\alpha} \bar{v}, \partial^{\alpha} \bar{E}\right\rangle \\
& -\kappa_{3} \sum_{1 \leq|\alpha| \leq N-2}\left\langle\nabla \times \partial^{\alpha} \bar{E}, \partial^{\alpha} \bar{B}\right\rangle .
\end{aligned}
$$

Similarly, one can choose $0<\kappa_{3} \ll \kappa_{2} \ll \kappa_{1} \ll 1$ with $\kappa_{2}^{3 / 2} \ll \kappa_{3}$ such that $\mathcal{E}_{N}^{h}(\bar{V}(t)) \sim$ $\|\nabla \bar{V}(t)\|_{N-1}^{2}$ because $\sigma_{s t}+\Phi\left(\sigma_{s t}\right)$ depends only on $x$ sufficiently small compared with 1 . Furthermore, the linear combination of previously obtained four estimates with coefficients corresponding to (4.13) yields (4.12) with $\mathcal{D}_{N}^{h}(\cdot)$ defined in (3.14). This completes the proof of Lemma 4.2, 
Now, we begin with the time-weighted estimate and iteration for the Lyapunov inequality (4.11). Let $\ell \geq 0$. Multiplying (4.11) by $(1+t)^{\ell}$ and taking integration over $[0, t]$ give

$$
\begin{aligned}
& (1+t)^{\ell} \mathcal{E}_{N}(\bar{V}(t))+\lambda \int_{0}^{t}(1+s)^{\ell} \mathcal{D}_{N}(\bar{V}(s)) d s \\
\leq & \mathcal{E}_{N}\left(\bar{V}_{0}\right)+\ell \int_{0}^{t}(1+s)^{\ell-1} \mathcal{E}_{N}(\bar{V}(s)) d s .
\end{aligned}
$$

Noticing

$$
\mathcal{E}_{N}(\bar{V}(t)) \leq C\left(D_{N+1}(\bar{V}(t))+\|\bar{B}\|^{2}\right),
$$

it follows that

$$
\begin{aligned}
& (1+t)^{\ell} \mathcal{E}_{N}(\bar{V}(t))+\lambda \int_{0}^{t}(1+s)^{\ell} \mathcal{D}_{N}(\bar{V}(s)) d s \\
\leq & \mathcal{E}_{N}\left(\bar{V}_{0}\right)+C \ell \int_{0}^{t}(1+s)^{\ell-1}\|\bar{B}(s)\|^{2} d s+C \ell \int_{0}^{t}(1+s)^{\ell-1} \mathcal{D}_{N+1}(\bar{V}(s)) d s .
\end{aligned}
$$

Similarly, it holds that

$$
\begin{aligned}
& (1+t)^{\ell-1} \mathcal{E}_{N+1}(\bar{V}(t))+\lambda \int_{0}^{t}(1+s)^{\ell-1} \mathcal{D}_{N+1}(\bar{V}(s)) d s \\
\leq & \mathcal{E}_{N+1}\left(\bar{V}_{0}\right)+C(\ell-1) \int_{0}^{t}(1+s)^{\ell-2}\|\bar{B}(s)\|^{2} d s+C(\ell-1) \int_{0}^{t}(1+s)^{\ell-2} \mathcal{D}_{N+2}(\bar{V}(s)) d s,
\end{aligned}
$$

and

$$
\mathcal{E}_{N+2}(\bar{V}(t))+\lambda \int_{0}^{t} \mathcal{D}_{N+2}(\bar{V}(s)) d s \leq \mathcal{E}_{N+2}\left(\bar{V}_{0}\right) .
$$

Then, for $1<\ell<2$, it follows by iterating the above estimates that

$$
\begin{aligned}
& (1+t)^{\ell} \mathcal{E}_{N}(\bar{V}(t))+\lambda \int_{0}^{t}(1+s)^{\ell} \mathcal{D}_{N}(\bar{V}(s)) d s \\
\leq & C \mathcal{E}_{N+2}\left(\bar{V}_{0}\right)+C \int_{0}^{t}(1+s)^{\ell-1}\|\bar{B}(s)\|^{2} d s .
\end{aligned}
$$

Similarly, for $2<\kappa<3$, the time-weighted estimate and iteration for the Lyapunov inequality (4.12) give

$$
\begin{aligned}
& (1+t)^{\kappa} \mathcal{E}_{N}^{h}(\bar{V}(t))+\lambda \int_{0}^{t}(1+s)^{\kappa} \mathcal{D}_{N}^{h}(\bar{V}(s)) d s \\
\leq & C \mathcal{E}_{N+3}^{h}\left(\bar{V}_{0}\right)+C \int_{0}^{t}(1+s)^{\kappa-1}\|\nabla \bar{B}(s)\|^{2} d s .
\end{aligned}
$$

Here the smallness of $\left\|n_{b}-1\right\|_{W_{0}^{N+4,2}}$ has been used in the process of iteration for the Lyapunov inequalities (4.11) and (4.12). Taking $\kappa=l+1$, it holds that

$$
\begin{aligned}
&(1+t)^{l+1} \mathcal{E}_{N}^{h}(\bar{V}(t))+\lambda \int_{0}^{t}(1+s)^{l+1} \mathcal{D}_{N}^{h}(\bar{V}(s)) d s \\
& \leq C \mathcal{E}_{N+3}^{h}\left(\bar{V}_{0}\right)+C \int_{0}^{t}(1+s)^{l}\|\nabla \bar{B}(s)\|^{2} d s \\
& \quad \leq C \mathcal{E}_{N+3}^{h}\left(\bar{V}_{0}\right)+C \int_{0}^{t}(1+s)^{\ell} \mathcal{D}_{N}(\bar{V}(s)) d s .
\end{aligned}
$$


Combining (4.14) with (4.15), we have

$$
\begin{aligned}
& (1+t)^{\ell} \mathcal{E}_{N}(\bar{V}(t))+\int_{0}^{t}(1+s)^{\ell} \mathcal{D}_{N}(\bar{V}(s)) d s \\
& +(1+t)^{l+1} \mathcal{E}_{N}^{h}(\bar{V}(t))+\int_{0}^{t}(1+s)^{l+1} \mathcal{D}_{N}^{h}(\bar{V}(s)) d s \\
& \quad \leq C \mathcal{E}_{N+3}\left(\bar{V}_{0}\right)+C \int_{0}^{t}(1+s)^{\ell-1}\|\bar{B}(s)\|^{2} d s .
\end{aligned}
$$

For this time, to estimate the integral term on the r.h.s. of (4.16), let's define

$$
\begin{gathered}
\mathcal{E}_{N, \infty}(\bar{V}(t))=\sup _{0 \leq s \leq t}\left\{(1+s)^{\frac{3}{2}} \mathcal{E}_{N}(\bar{V}(s))+(1+s)^{\frac{5}{2}} \mathcal{E}_{N}^{h}(\bar{V}(s))\right\}, \\
L_{0}(t)=\sup _{0 \leq s \leq t}(1+s)^{\frac{5}{2}}\|[\bar{\rho}, \bar{u}]\|^{2} .
\end{gathered}
$$

Then, we have the following

Lemma 4.3. For any $t \geq 0$, it holds that:

$$
\begin{aligned}
\|\bar{B}(t)\|^{2} \leq C(1+t)^{-\frac{3}{2}}\left(\left\|\left[\bar{\sigma}_{0}, \bar{v}_{0}\right]\right\|^{2}+\left\|\left[\bar{v}_{0}, \bar{E}_{0}, \bar{B}_{0}\right]\right\|_{L^{1} \cap \dot{H}^{2}}^{2}\right. \\
\left.+\left[\mathcal{E}_{N, \infty}(\bar{V}(t))\right]^{2}+\delta^{2} \mathcal{E}_{N, \infty}(\bar{V}(t))\right) .
\end{aligned}
$$

Proof. Applying the fourth linear estimate on $B$ in (4.7) to the mild form (4.10) gives

$$
\begin{aligned}
\left\|B_{1}(t)\right\| \leq C(1+t)^{-\frac{3}{4}} \|\left[\bar{u}_{0},\right. & \left.E_{1,0}, B_{1,0}\right] \|_{L^{1} \cap \dot{H}^{2}} \\
& +C \int_{0}^{t}(1+t-s)^{-\frac{3}{4}}\left\|\left[g_{2}(s), g_{3}(s)\right]\right\|_{L^{1} \cap \dot{H}^{2}} d s .
\end{aligned}
$$

Applying the $L^{2}$ linear estimate on $u$ in (4.7) to the mild form (4.10),

$$
\begin{aligned}
\|\bar{u}(t)\| \leq C(1+t)^{-\frac{5}{4}}( & \left.\left\|\bar{\rho}_{0}\right\|+\left\|\left[\bar{u}_{0}, E_{1,0}, B_{1,0}\right]\right\|_{L^{1} \cap \dot{H}^{2}}\right) \\
& +C \int_{0}^{t}(1+t-s)^{-\frac{5}{4}}\left(\left\|g_{1}(s)\right\|+\left\|\left[g_{2}(s), g_{3}(s)\right]\right\|_{L^{1} \cap \dot{H}^{2}}\right) d s .
\end{aligned}
$$

Applying the $L^{2}$ linear estimate on $\rho$ in (4.7) to (4.10), one has

$$
\|\bar{\rho}(t)\| \leq C e^{-\frac{t}{2}}\left\|\left[\bar{\rho}_{0}, \bar{u}_{0}\right]\right\|+C \int_{0}^{t} e^{-\frac{t-s}{2}}\left\|\left[g_{1}(s), g_{2}(s)\right]\right\| d s .
$$

Recall the definition (4.4) of $g_{1}, g_{2}$ and $g_{3}$,

$$
\begin{aligned}
& g_{1}(s)=-\rho_{s t} \nabla \cdot \bar{u}-\bar{\rho} \nabla \cdot \bar{u}-\bar{u} \cdot \nabla \rho_{s t}-\bar{u} \cdot \nabla \bar{\rho}, \\
& g_{2}(s) \sim \bar{u} \cdot \nabla \bar{u}+\bar{u} \times B_{1}+\bar{\rho} \nabla \bar{\rho}+\rho_{s t} \nabla \bar{\rho}+\bar{\rho} \nabla \rho_{s t}, \\
& g_{3}(s)=\bar{\rho} \bar{u}+\rho_{s t} \bar{u} .
\end{aligned}
$$

Firstly, we estimate those terms including $\rho_{s t}$. It follows that

$$
\begin{aligned}
& \left\|\rho_{s t} \nabla \cdot \bar{u}\right\| \leq\left\|\rho_{s t}\right\|_{L^{\infty}}\|\nabla \bar{u}\|, \quad\left\|\bar{u} \cdot \nabla \rho_{s t}\right\| \leq\left\|\nabla \rho_{s t}\right\|\|\bar{u}\|_{L^{\infty}} \leq\left\|\nabla \rho_{s t}\right\|\|\nabla \bar{u}\|_{H^{1}}, \\
& \left\|\rho_{s t} \nabla \bar{\rho}\right\|_{L^{1}} \leq\left\|\rho_{s t}\right\|\|\nabla \bar{\rho}\|, \quad\left\|\rho_{s t} \nabla \bar{\rho}\right\| \leq\left\|\rho_{s t}\right\|\left\|_{L^{\infty}}\right\| \nabla \bar{\rho}\|\leq\| \nabla \rho_{s t}\left\|_{H^{1}}\right\| \nabla \bar{\rho} \|, \\
& \left\|\bar{\rho} \nabla \rho_{s t}\right\|_{L^{1}} \leq\left\|\nabla \rho_{s t}\right\|\|\bar{\rho}\|, \quad\left\|\nabla \rho_{s t} \bar{\rho}\right\| \leq\|\bar{\rho}\|_{L^{\infty}}\left\|\rho_{s t}\right\| \leq\left\|\rho_{s t}\right\|\|\nabla \bar{\rho}\|_{H^{1}}, \\
& \left\|\rho_{s t} \bar{u}\right\|_{L^{1}} \leq\left\|\rho_{s t}\right\|\|\bar{u}\|,
\end{aligned}
$$


and for $|\alpha|=2$, one has

$$
\begin{aligned}
\left\|\partial^{\alpha}\left(\rho_{s t} \nabla \bar{\rho}\right)\right\| & \leq\left\|\rho_{s t} \partial^{\alpha} \nabla \bar{\rho}\right\|+\left\|\partial^{\alpha}\left(\rho_{s t} \nabla \bar{\rho}\right)-\rho_{s t} \partial^{\alpha} \nabla \bar{\rho}\right\| \\
& \leq\left\|\rho_{s t}\right\|_{L^{\infty}}\left\|\partial^{\alpha} \nabla \bar{\rho}\right\|+C\left\|\nabla \rho_{s t}\right\|_{H^{|\alpha|-1}}\|\nabla \bar{\rho}\|_{L^{\infty}}+C\left\|\nabla \rho_{s t}\right\|_{L^{\infty}}\|\nabla \bar{\rho}\|_{H^{|\alpha|-1}} \\
& \leq C \delta\|\nabla \bar{\rho}\|_{H^{2}}
\end{aligned}
$$

where we have used the estimate $\left\|\partial^{\alpha}(f g)-f \partial^{\alpha} g\right\| \leq C\|\nabla f\|_{H^{k-1}}\|g\|_{L^{\infty}}+C\|\nabla f\|_{L^{\infty}}\|g\|_{H^{k-1}}$, for any $|\alpha|=k$. Similarly, it holds that

$$
\begin{gathered}
\left\|\partial^{\alpha}\left(\rho_{s t} \bar{u}\right)\right\| \leq\left\|\bar{u} \partial^{\alpha} \rho_{s t}\right\|+\left\|\partial^{\alpha}\left(\rho_{s t} \bar{u}\right)-\bar{u} \partial^{\alpha} \rho_{s t}\right\| \leq C \delta\|\nabla \bar{u}\|_{H^{2}}, \\
\left\|\partial^{\alpha}\left(\bar{\rho} \nabla \rho_{s t}\right)\right\| \leq C \delta\|\nabla \bar{\rho}\|_{H^{2}} .
\end{gathered}
$$

It is straightforward to verify that for any $0 \leq s \leq t$,

$$
\begin{gathered}
\left\|\left[g_{2}(s), g_{3}(s)\right]\right\|_{L^{1}} \leq C\|\bar{u}\|\|\nabla \bar{u}\|+\|\bar{u}\|\left\|B_{1}\right\|+\|\bar{\rho}\|\|\bar{u}\|+\|\bar{\rho}\|\|\nabla \bar{\rho}\| \\
+C\left(\left\|\rho_{s t} \nabla \bar{\rho}\right\|_{L^{1}}+\left\|\rho_{s t} \bar{u}\right\|_{L^{1}}+\left\|\bar{\rho} \nabla \rho_{s t}\right\|_{L^{1}}\right) \\
\leq C \mathcal{E}_{N}(\bar{U}(s))+C \delta \sqrt{\mathcal{E}_{N}^{h}(\bar{U}(s))}+C \delta\|[\bar{\rho}, \bar{u}]\| \\
\left\|\left[g_{2}(s), g_{3}(s)\right]\right\|_{\dot{H}^{2}} \leq C \mathcal{E}_{N}(\bar{U}(s))+C \delta \sqrt{\mathcal{E}_{N}^{h}(\bar{U}(s))}
\end{gathered}
$$

and

$$
\left\|\left[g_{1}(s), g_{2}(s)\right]\right\| \leq C \mathcal{E}_{N}(\bar{U}(s))+C \delta \sqrt{\mathcal{E}_{N}^{h}(\bar{U}(s))} .
$$

Notice that $\mathcal{E}_{N}(\bar{U}(s)) \leq C \mathcal{E}_{N}(\bar{V}(\sqrt{\gamma} s))$. From (4.17) and (4.18), for any $0 \leq s \leq t$,

$$
\begin{gathered}
\mathcal{E}_{N}(\bar{V}(\sqrt{\gamma} s)) \leq(1+\sqrt{\gamma} s)^{-\frac{3}{2}} \mathcal{E}_{N, \infty}(\bar{V}(\sqrt{\gamma} t)), \\
\mathcal{E}_{N}^{h}(\bar{V}(\sqrt{\gamma} s)) \leq(1+\sqrt{\gamma} s)^{-\frac{5}{2}} \mathcal{E}_{N, \infty}(\bar{V}(\sqrt{\gamma} t)), \\
\|[\bar{\rho}, \bar{u}](s)\| \leq \sqrt{L_{0}(t)}(1+s)^{-\frac{5}{4}}
\end{gathered}
$$

Then, it follows that for $0 \leq s \leq t$,

$$
\begin{aligned}
\left\|\left[g_{2}(s), g_{3}(s)\right]\right\|_{L^{1}} \leq C(1+\sqrt{\gamma} s)^{-\frac{3}{2}} & \mathcal{E}_{N, \infty}(\bar{V}(\sqrt{\gamma} t)) \\
+ & C \delta(1+\sqrt{\gamma} s)^{-\frac{5}{4}} \sqrt{\mathcal{E}_{N, \infty}(\bar{V}(\sqrt{\gamma} t))}+C \delta \sqrt{L_{0}(t)}(1+s)^{-\frac{5}{4}} \\
\left\|\left[g_{2}(s), g_{3}(s)\right]\right\|_{\dot{H}^{2}} \leq & C(1+\sqrt{\gamma} s)^{-\frac{3}{2}} \mathcal{E}_{N, \infty}(V(\sqrt{\gamma} t)) \\
& +C \delta(1+\sqrt{\gamma} s)^{-\frac{5}{4}} \sqrt{\mathcal{E}_{N, \infty}(\bar{V}(\sqrt{\gamma} t))} \\
\left\|\left[g_{1}(s), g_{2}(s)\right]\right\| \leq & C(1+\sqrt{\gamma} s)^{-\frac{3}{2}} \mathcal{E}_{N, \infty}(V(\sqrt{\gamma} t)) \\
& +C \delta(1+\sqrt{\gamma} s)^{-\frac{5}{4}} \sqrt{\mathcal{E}_{N, \infty}(\bar{V}(\sqrt{\gamma} t))}
\end{aligned}
$$

Putting the above inequalities into (4.20), (4.21) and (4.22) respectively gives

$$
\begin{aligned}
& \left\|B_{1}(t)\right\| \leq C(1+t)^{-\frac{3}{4}}\left\{\left\|\left[\bar{u}_{0}, E_{1,0}, B_{1,0}\right]\right\|_{L^{1} \cap \dot{H}^{2}}+\mathcal{E}_{N, \infty}(\bar{V}(\sqrt{\gamma} t))\right. \\
& \left.+\delta \sqrt{L_{0}(t)}+\delta \sqrt{\mathcal{E}_{N, \infty}(\bar{V}(\sqrt{\gamma} t))}\right\}
\end{aligned}
$$




$$
\begin{aligned}
& \|\bar{u}(t)\| \leq C(1+t)^{-\frac{5}{4}}\left\{\left\|\bar{\rho}_{0}\right\|+\left\|\left[\bar{u}_{0}, E_{1,0}, B_{1,0}\right]\right\|_{L^{1} \cap \dot{H}^{2}}+\mathcal{E}_{N, \infty}(\bar{V}(\sqrt{\gamma} t))\right. \\
& \left.+\delta \sqrt{L_{0}(t)}+\delta \sqrt{\mathcal{E}_{N, \infty}(\bar{V}(\sqrt{\gamma} t))}\right\} \\
& \|\bar{\rho}(t)\| \leq C(1+t)^{-\frac{5}{4}}\left\{\left\|\left[\bar{\rho}_{0}, u_{0}\right]\right\|+\mathcal{E}_{N, \infty}(\bar{V}(\sqrt{\gamma} t))\right. \\
& \left.+\delta \sqrt{\mathcal{E}_{N, \infty}(\bar{V}(\sqrt{\gamma} t))}\right\} .
\end{aligned}
$$

The definition of $L_{0}(t)$, (4.27) and (4.28) further imply that

$$
\begin{aligned}
L_{0}(t) \leq C\left\|\left[\bar{\rho}_{0}, u_{0}\right]\right\|^{2}+C \|\left[\bar{u}_{0}, E_{1,0}, B_{1,0}\right] & \|_{L^{1} \cap \dot{H}^{2}}^{2} \\
& +C\left[\mathcal{E}_{N, \infty}(\bar{V}(\sqrt{\gamma} t))\right]^{2}+C \delta^{2} \mathcal{E}_{N, \infty}(\bar{V}(\sqrt{\gamma} t)),
\end{aligned}
$$

where we have used that $\delta$ is small enough. Plugging the above estimate into (4.26) implies (4.19), since $\|\bar{B}(t)\| \leq C\left\|B_{1}(t / \sqrt{\gamma})\right\|$ and $\left[\bar{\rho}, \bar{u}, E_{1}, B_{1}\right]$ is equivalent with $[\bar{\sigma}, \bar{v}, \bar{E}, \bar{B}]$ up to a positive constant. This completes the proof of Lemma 4.3.

Now, the rest is to prove the uniform-in-time bound of $\mathcal{E}_{N, \infty}(\bar{V}(t))$ which yields the timedecay rates of the Lyapunov functionals $\mathcal{E}_{N}(\bar{V}(t))$ and $\mathcal{E}_{N}^{h}(\bar{V}(t))$ thus $\|\bar{V}(t)\|_{N}^{2},\|\nabla \bar{V}(t)\|_{N-1}^{2}$. In fact, by taking $\ell=\frac{3}{2}+\epsilon$ in (4.16) with $\epsilon>0$ small enough, one has

$$
\begin{aligned}
&(1+t)^{\frac{3}{2}+\epsilon} \mathcal{E}_{N}(\bar{V}(t))+ \int_{0}^{t}(1+s)^{\frac{3}{2}+\epsilon} \mathcal{D}_{N}(\bar{V}(s)) d s \\
&+(1+t)^{\frac{5}{2}+\epsilon} \mathcal{E}_{N}^{h}(\bar{V}(t))+\int_{0}^{t}(1+s)^{\frac{5}{2}+\epsilon} \mathcal{D}_{N}^{h}(\bar{V}(s)) d s \\
& \quad \leq C \mathcal{E}_{N+3}\left(\bar{V}_{0}\right)+C \int_{0}^{t}(1+s)^{\frac{1}{2}+\epsilon}\|\bar{B}(s)\|^{2} d s .
\end{aligned}
$$

Here, using (4.19) and the fact $\mathcal{E}_{N, \infty}(\bar{V}(t))$ is non-decreasing in $t$, it further holds that

$$
\begin{array}{r}
\int_{0}^{t}(1+s)^{\frac{1}{2}+\epsilon}\|\bar{B}(s)\|^{2} d s \leq C(1+t)^{\epsilon}\left\{\left\|\left[\bar{\sigma}_{0}, \bar{v}_{0}\right]\right\|^{2}+\left\|\left[\bar{v}_{0}, \bar{E}_{0}, \bar{B}_{0}\right]\right\|_{L^{1} \cap \dot{H}^{2}}^{2}\right. \\
\left.+\left[\mathcal{E}_{N, \infty}(\bar{V}(t))\right]^{2}+\delta^{2} \mathcal{E}_{N, \infty}(\bar{V}(t))\right\} .
\end{array}
$$

Therefore, it follows that

$$
\begin{aligned}
&(1+t)^{\frac{3}{2}+\epsilon} \mathcal{E}_{N}(\bar{V}(t))+(1+t)^{\frac{5}{2}+\epsilon} \mathcal{E}_{N}^{h}(\bar{V}(t)) \\
& \quad+\int_{0}^{t}(1+s)^{\frac{3}{2}+\epsilon} \mathcal{D}_{N}(\bar{V}(s)) d s+\int_{0}^{t}(1+s)^{\frac{5}{2}+\epsilon} \mathcal{D}_{N}^{h}(\bar{V}(s)) d s \\
& \leq C \mathcal{E}_{N+3}\left(\bar{V}_{0}\right)+C(1+t)^{\epsilon}\left(\left\|\left[\bar{\sigma}_{0}, \bar{v}_{0}\right]\right\|^{2}+\left\|\left[\bar{v}_{0}, \bar{E}_{0}, \bar{B}_{0}\right]\right\|_{L^{1} \cap \dot{H}^{2}}^{2}\right. \\
&+ \\
&\left.+\left[\mathcal{E}_{N, \infty}(\bar{V}(t))\right]^{2}+\delta^{2} \mathcal{E}_{N, \infty}(\bar{V}(t))\right),
\end{aligned}
$$

which implies

$$
\begin{aligned}
(1+t)^{\frac{3}{2}} \mathcal{E}_{N}(\bar{V}(t))+(1+t)^{\frac{5}{2}} \mathcal{E}_{N}^{h}(\bar{V}(t)) \leq C\left\{\mathcal{E}_{N+3}\left(\bar{V}_{0}\right)\right. & +\left\|\left[\bar{v}_{0}, \bar{E}_{0}, \bar{B}_{0}\right]\right\|_{L^{1}}^{2} \\
& \left.+\left[\mathcal{E}_{N, \infty}(\bar{V}(t))\right]^{2}+\delta^{2} \mathcal{E}_{N, \infty}(\bar{V}(t))\right\},
\end{aligned}
$$

and thus

$$
\mathcal{E}_{N, \infty}(\bar{V}(t)) \leq C\left(\epsilon_{N+3}\left(\bar{V}_{0}\right)^{2}+\mathcal{E}_{N, \infty}(\bar{V}(t))^{2}\right)
$$


Here, we have used that $\delta$ is small enough. Recall the definition of $\epsilon_{N+3}\left(\bar{V}_{0}\right)$, since $\epsilon_{N+3}\left(\bar{V}_{0}\right)>$ 0 is sufficiently small, $\mathcal{E}_{N, \infty}(\bar{V}(t)) \leq C \epsilon_{N+3}\left(\bar{V}_{0}\right)^{2}$ holds true for any $t \geq 0$, which implies

$$
\begin{gathered}
\|\bar{V}(t)\|_{N} \leq C \mathcal{E}_{N}(\bar{V}(t))^{1 / 2} \leq C \epsilon_{N+3}\left(\bar{V}_{0}\right)(1+t)^{-\frac{3}{4}}, \\
\|\nabla \bar{V}(t)\|_{N-1} \leq C \mathcal{E}_{N}^{h}(\bar{V}(t))^{1 / 2} \leq C \epsilon_{N+3}\left(\bar{V}_{0}\right)(1+t)^{-\frac{5}{4}} .
\end{gathered}
$$

The definition of $L_{0}(t)$, the uniform-in-time bound of $\mathcal{E}_{N, \infty}(\bar{V}(t))$ and (4.29) show that

$$
\|[\bar{\rho}, \bar{u}](t)\| \leq C \epsilon_{N+3}\left(\bar{V}_{0}\right)(1+t)^{-\frac{5}{4}} .
$$

In addition, applying the $L^{2}$ linear estimate on $E$ in (4.7) to the mild form (4.10),

$$
\begin{aligned}
\left\|E_{1}(t)\right\| \leq C(1+t)^{-\frac{5}{4}}\left\|\left[\bar{u}_{0}, E_{1,0}, B_{1,0}\right]\right\|_{L^{1} \cap \dot{H}^{3}} \\
\\
\quad+C \int_{0}^{t}(1+t-s)^{-\frac{5}{4}}\left\|\left[g_{2}(s), g_{3}(s)\right]\right\|_{L^{1} \cap \dot{H}^{3}} d s .
\end{aligned}
$$

Since by (4.31) and (4.32), similar to obtaining (4.23) and (4.24), we have

$$
\left\|\left[g_{2}(s), g_{3}(s)\right]\right\|_{L^{1} \cap \dot{H}^{3}} \leq C\|\bar{U}(t)\|_{4}^{2}+C \delta\|\nabla \bar{U}(t)\|_{3}+C \delta\|[\bar{\rho}, \bar{u}]\| \leq C \epsilon_{7}\left(\bar{V}_{0}\right)(1+t)^{-\frac{5}{4}},
$$

it follows that

$$
\left\|E_{1}(t)\right\| \leq C \epsilon_{7}\left(\bar{V}_{0}\right)(1+t)^{-\frac{5}{4}} .
$$

This completes Theorem 1.2 .

Acknowledgements: The first author Qingqing Liu would like to thank Dr. Renjun Duan for his guidance and continuous help. The research was supported by the National Natural Science Foundation of China \#11071093, the PhD specialized grant of the Ministry of Education of China \#20100144110001, and the Special Fund for Basic Scientific Research of Central Colleges \#CCNU10C01001, \#CCNU12C01001.

\section{REFERENCES}

[1] C. Besse, J. Claudel, P. Degond, et al., A model hierarchy for ionospheric plasma modeling, Math. Models Methods Appl. Sci., 14(2004), 393-415.

[2] D. Chae and E. Tadmor, On the finite time blow-up of the Euler-Poisson equations in $\mathbb{R}^{n}$, Commun. Math. Sci., 6(2008), 785-789.

[3] G.Q. Chen, J.W. Jerome and D.H. Wang, Compressible Euler-Maxwell equations, Transport Theory Statist. Phys., 29(2000), 311-331.

[4] Y.B. Deng, T.P. Liu, T. Yang and Z.A. Yao, Solutions of Euler-Poisson equations for gaseous stars, Arch. Ration. Mech. Anal., 164(2002), 261-285.

[5] R.J. Duan, Global smooth flows for the compressible Euler-Maxwell system. The relaxation case. J. Hyperbolic Differ. Equ., 8(2011), 375-413.

[6] R.J. Duan, H.X. Liu, S. Ukai and T. Yang, Optimal $L^{p}-L^{q}$ convergence rates for the compressible NavierStokes equations with potential force, J. Differential Equations, 238(2007), 220-233.

[7] R.J. Duan, Q.Q. Liu, C.J. Zhu, The Cauchy problem on the compressible two-fluids Euler-Maxwell equations, SIAM J. Math. Anal., 44(2012), 102-133.

[8] R.J. Duan and R.M. Strain, Optimal time decay of the Vlasov-Poisson-Boltzmann system in $\mathbb{R}^{3}$, Arch. Ration. Mech. Anal., 199(2011), 291-328.

[9] R.J. Duan, S. Ukai and T. Yang, A combination of energy method and spectral analysis for study of equations of gas motion, Front. Math. China, 4(2009), 253-282.

[10] R.J. Duan, S. Ukai, T. Yang and H.J Zhao, Optimal convergence rates for the compressible Navier-Stokes equations with potential forces, Math. Models Methods Appl. Sci., 17(2007), 737-758.

[11] R.J. Duan and T. Yang, Stability of the one-species Vlasov-Poisson-Boltzmann system, SIAM J. Math. Anal., 41(2009/10), 2353-2387.

[12] Y. Guo, Smooth irrotational flows in the large to the Euler-Poisson system in $\mathbb{R}^{3+1}$, Comm. Math. Phys., 195(1998), 249-265.

[13] Y. Guo, B. Pausader, Global smooth ion dynamics in the Euler-Poisson system, Comm. Math. Phys., 303(2011), 89-125. 
[14] M.L. Hajjej, Y.J. Peng, Initial layers and zero-relaxation limits of Euler-Maxwell equations, J. Differential Equations, 252(2012), 1441-1465.

[15] J.W. Jerome, The Cauchy problem for compressible hydrodynamic-Maxwell systems: a local theory for smooth solutions, Differential Integral Equations, 16(2003), 1345-1368.

[16] T. Kato, The Cauchy problem for quasi-linear symmetric hyperbolic systems, Arch. Rational Mech. Anal., 58(1975), 181-205.

[17] T. Luo, R. Natalini and Z.P. Xin, Large time behavior of the solutions to a hydrodynamic model for semiconductors, SIAM J. Appl. Math., 59(1999), 810-830.

[18] T. Luo and J. Smoller, Existence and non-linear stability of rotating star solutions of the compressible Euler-Poisson equations, Arch. Ration. Mech. Anal., 191(2009), 447-496.

[19] Y.J. Peng and S. Wang, Convergence of compressible Euler-Maxwell equations to incompressible Euler equations, Comm. Partial Differential Equations, 33(2008), 349-376.

[20] Y.J. Peng, S. Wang, Convergence of compressible Euler-Maxwell equations to compressible Euler-Poisson equations, Chin. Ann. Math. Ser. B, 28(2007), 583-602.

[21] Y.J. Peng, S. Wang, Rigorous derivation of incompressible e-MHD equations from compressible EulerMaxwell equations, SIAM J. Math. Anal., 40(2008) 540-565.

[22] H. Rishbeth and O.K. Garriott, Introduction to Ionospheric Physics, Academic Press, 1969.

[23] E.M. Stein, Singular Integrals and Differentiability Properties of Functions, Princeton Mathematical Series, No. 30 Princeton University Press, Princeton, N.J. 1970 xiv +290 pp.

[24] M.E. Taylor, Partial Differential Equations, I. Basic Theory, Springer, New York, 1996.

[25] Y. Ueda and S. Kawashima, Decay property of regularity-loss type for the Euler-Maxwell system, Methods Appl. Anal., 18(2011), 245-268.

[26] Y. Ueda, S. Wang and S. Kawashima, Dissipative Structure of the Regularity-Loss Type and Time Asymptotic Decay of Solutions for the Euler-Maxwell System, SIAM J. Math. Anal., 44(2012), 20022017.

(QQL) The Hubei Key Laboratory of Mathematical Physics, School of Mathematics and Statistics, Central China Normal University, Wuhan, 430079, P. R. China

E-mail address: shuxueliuqingqing@126.com

(CJZ) The Hubei Key Laboratory of Mathematical Physics, School of Mathematics and Statistics, Central China Normal University, Wuhan, 430079, P. R. China

E-mail address: cjzhu@mail.ccnu.edu.cn 